Classification

Physics Abstracts

$7.130-7.221-8.814$

\title{
MECHANICAL INSTABILITIES OF SMECTIC-A LIQUID CRYSTALS UNDER DILATIVE OR COMPRESSIVE STRESSES
}

\author{
R. RIBOTTA and G. DURAND \\ Laboratoire de Physique des Solides (*), Université Paris-Sud \\ 91405 Orsay, France
}

(Reçu le 30 août 1976, accepté le 4 novembre 1976)

\begin{abstract}
Résumé. - Un modèle utilisant l'élasticité de type mixte introduite par de Gennes est construit pour décrire le comportement de cristaux liquides smectiques $\mathrm{A}$ soumis à des forces de compression ou de dilatation normales aux couches.

Ce modèle prévoit deux types d'instabilités mécaniques, respectivement : basculement des molécules dans les couches et ondulation des couches. On donne les expressions pour les seuils d'instabilités et l'amplitude des déformations produites. Les résultats expérimentaux sont présentés qui confirment ces prédictions et donnent des mesures de la rigidité $\left(B_{\perp}\right)$ des couches relativement au basculement des molécules, et de la longueur de pénétration $\lambda$ de de Gennes. On observe en outre une dépendance en temps de l'amplitude de la déformation qui est expliquée par un processus de relaxation de la contrainte appliquée, dû au mouvement de dislocations-coin. La variation des seuils en fonction de la température est étudiée dans des corps qui présentent des transitions de quasi second ordre vers les phases nématiques ou smectiques $\mathrm{C}$. Au voisinage d'une phase nématique le seuil d'instabilité d'ondulation, et donc $\lambda$, diverge comme prévu, mais avec un exposant critique apparent 0,16 très inférieur à la valeur prévue pour l'exposant $v / 2$ (on s'attend à 0,25 dans un modèle de champ moyen et à 0,33 dans un modèle non classique de type transition $\lambda$ de l'hélium). Ce large désaccord n'est pas expliqué encore. Au voisinage d'une phase smectique-C le module de rigidité $B_{\perp}$ s'annule avec un exposant critique apparent de type classique $\gamma \simeq 1$.
\end{abstract}

\begin{abstract}
Using the mixed-type elasticity for smectics introduced by de Gennes a model is built to describe the behaviour of homeotropic smectic-A liquid crystals submitted to compressive or dilative forces normal to the layers. This model predicts respectively two types of mechanical instabilities : molecular tilt inside the layers, or undulation of the layers. The expressions for the thresholds of these instabilities and the amplitude of the deformations produced are given. Experimental results are presented which confirm these predictions and give measurements of the rigidity $B_{\perp}$ of the layers compared to the molecular tilt and of the penetration length $\lambda$ of de Gennes. In addition the time dependence of the instabilities is observed and is explained in term of the relaxation of the applied stress due to the motion ( $\mathrm{climb}$ ) of edge-dislocations. The temperature dependence of the instability thresholds is measured in materials presenting quasi-second order transitions towards nematic or smectic-C phases. Close to a nematic phase the dilative instability threshold and thus $\lambda$ diverge as expected, but with an apparent critical exponent $(0.16)$, significatively smaller than the expected exponent $v / 2$ ( 0.25 or 0.33 in a mean-field or in a non-classical model respectively). This discrepancy has not been explained yet. Close to a smectic-C phase the rigidity modulus $B_{\perp}$ vanishes with an apparent classical exponent $\gamma \simeq 1$.
\end{abstract}

1. Introduction. - Smectic-A liquid crystals are layered materials, with the molecules normal to the layers. They have been shown to exhibit a mechanical instability when dilative stresses are applied normal to the layers [1, 2]. Above a critical displacement of the boundary plates of an homeotropic sample, the layers undulate in order to compensate the increase of the sample thickness. It has also been shown [3] that in a

(*) Laboratoire associé au C.N.R.S. smectic A phase, an external compression can induce the $\mathrm{C}$ phase (the molecules tilt inside the layers). This effect is easier to observe close to a smectic-A to smectic-C transition. In this paper we first give a theoretical model of these mechanical instabilities induced in smectic-A liquid crystals : undulation instability of layers under dilative stress, and tilt instability of molecules inside the layers under compressive stress. For this model we use de Gennes' formulation of the elasticity for smectics [4]. We then 
present detailed measurements of the thresholds and relaxation times which complete the previously published letters $[1,3,11,13,14,15]$. We measure the observed temperature dependence of the thresholds close to nematic and smectic- $\mathrm{C}$ phases in materials presenting quasi-second-order phase transitions. We analyze these variations in term of critical behaviour of the elastic properties of the materials.

2. Theoretical treatment. -2.1 GENERAL DESCRIPTION OF THE MODEL. - We assume a homeotropic smectic-A sample (Fig. 1) with the layers in the $x O y$ plane. We follow the notations of ref. [1]. The sample thickness is $d$, the uniform displacement of the upper plate is $\delta$ (the lower plate is at rest). For dilations $\delta$ is positive while it is negative for compressions. The non-uniform displacement of the layers which may occur in the presence of the uniform external strain $X=\delta / d$ is called $u(x, y, z) ; \partial u / \partial x=\theta$ is the tilt angle of the layer with respect to the $x$ axis (see Fig. $1 b$ ). We

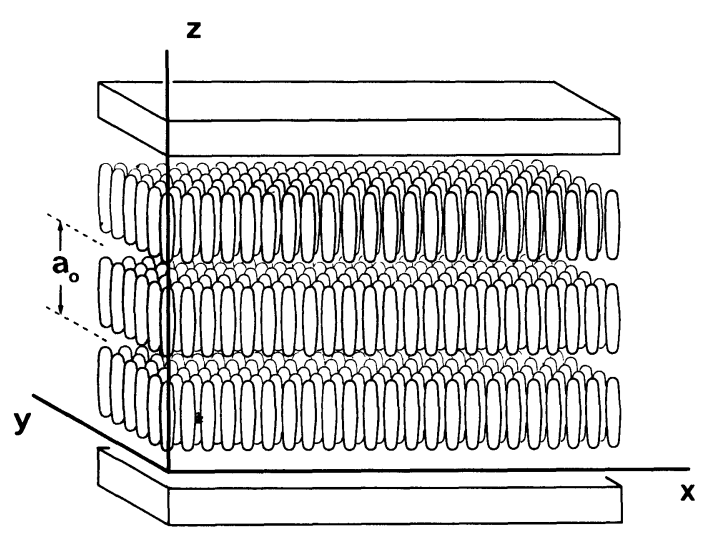

a)

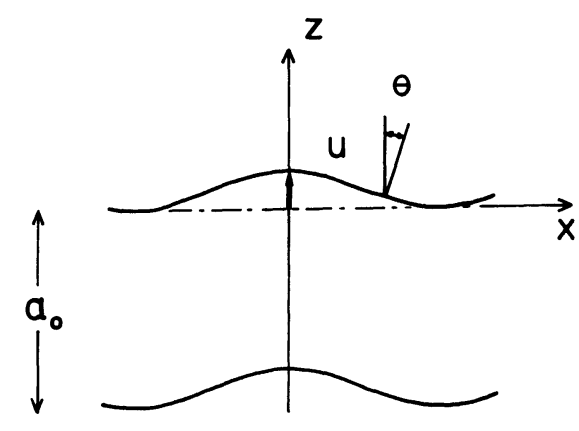

b)

FIG. 1. - a) Molecular arrangement of a smectic-A liquid crystal (the molecules are sketched as ellipsoids). In the homeotropic configuration the molecules are normal to the boundary surfaces (generally glass plates). $b$ ) Deformation of a layer. The variable $u$ is measured along $z$. The local bend angle $\theta=\partial u / \partial x$.

restrict ourselves for simplicity to a two-dimensional problem and call $\varphi$ a possible tilt angle of the molecular axis with respect to the normal to the layers; the director $\mathbf{n}$ is then at an angle $(\theta+\varphi)$ away from $O z$.
The contribution to the free-energy density, associated with a general strain $\partial u / \partial z$ is :

$$
\frac{1}{2} B\left(\frac{\partial u}{\partial z}\right)^{2} \equiv \frac{1}{2}\left[K_{1} / \lambda^{2}\right]\left(\frac{\partial u}{\partial z}\right)^{2}
$$

where $B$ is the isothermal normal compression modulus of the smectic layers $\left(B \simeq 10^{7}\right.$ dyne $\left./ \mathrm{cm}^{2}\right)$, $K_{1}$ is the Frank elastic constant associated with the splay deformation $\left(K_{1} \simeq 10^{-6}\right.$ dyne $)$ and $\lambda$ is the characteristic length defined by de Gennes $\left(\lambda=\left(K_{1} / B\right)^{1 / 2} \sim\right.$ molecular length $\left.a=20 \AA\right)$. The contribution to the free-energy density, associated with the molecular tilt $\varphi$ is included in ref. [5]; we write it :

$$
\frac{1}{2} B_{\perp} \varphi^{2} \equiv \frac{1}{2}\left(K_{1} / \lambda_{\perp}^{2}\right) \cdot \varphi^{2}
$$

for small values of $\varphi$.

$B_{\perp}$ is expected to be comparable to $B$ or smaller ; $\lambda_{\perp}$ is then of the order of $\lambda$ or larger. Close to a smectic $\mathrm{A} \leftrightarrow$ nematic second-order phase transition, $\lambda_{\perp}$ and $\lambda$ diverge, but their ratio is expected to remain constant. $\left(\lambda_{1}^{2} / \lambda^{2} \sim\right.$ (molecular length/molecular width $)^{2} \simeq 16$.) On the other hand, close to a second order $\mathrm{A} \leftrightarrow \mathrm{C}$ phase transition, $B$ is expected to remain constant while $B_{\perp}$ vanishes $[5,26]$ as $\left(T-T_{\mathrm{c}}\right)^{\gamma}$, since $\frac{1}{2} B_{\perp} \varphi^{2}$ is the first term of a Landau-like form of the free-energy density for the $\mathrm{C}$ phase expressed in terms of the square of the modulus $\varphi^{2}$ of its (tilt) order parameter $\varphi$; in a molecular field approximation $\gamma=1$ and $\gamma_{\perp}^{2}$ is expected to diverge accordingly at $T_{\mathrm{c}}$, assuming that $K_{1}$ also remains constant.

In order to describe mechanical instabilities, we need a non-linear term in the free energy. We write it by extending an argument from de Gennes : in the presence of molecular tilt $\varphi$, the normal layer thickness is $a_{0} \cdot \cos \varphi$ if we assume rigid molecules of length $a_{0}$ (Fig. 2b). When the layers are also tilted (Fig. 2b), their thickness measured along $\mathrm{Oz}$ becomes :

$$
a=a_{0} \cdot \frac{\cos \varphi}{\cos \theta} .
$$

We assume that the total sample thickness $d$ remains constant, and that the total number of layers is conserved (this assumption is correct at least in a transient regime, since creation or annihilation of layers is a relatively slow process related to permeation). The combined molecular tilt and layer tilt results in a strain

$$
(1-\cos \varphi / \cos \theta) \sim\left(\frac{\varphi^{2}}{2}-\frac{\theta^{2}}{2}\right)
$$

for small angles. The compression term $\left(\theta^{2} / 2\right)$ leads to a layer undulation instability under dilative stress; the dilation term $\left(\varphi^{2} / 2\right)$ is expected to give rise to a molecular tilt instability inside the layers, under 


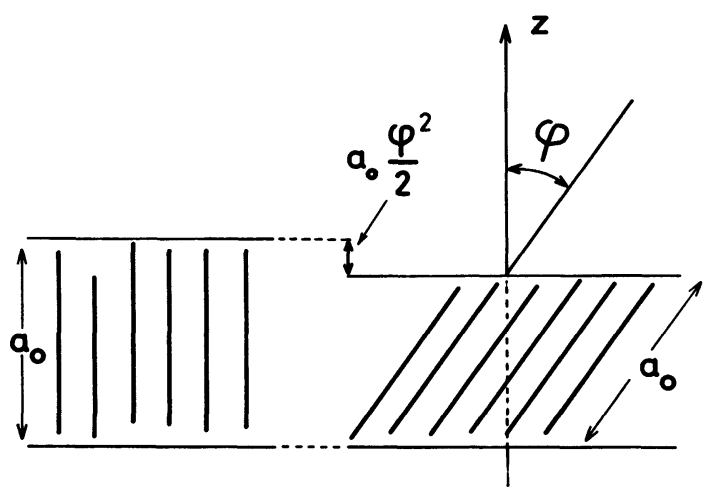

a)

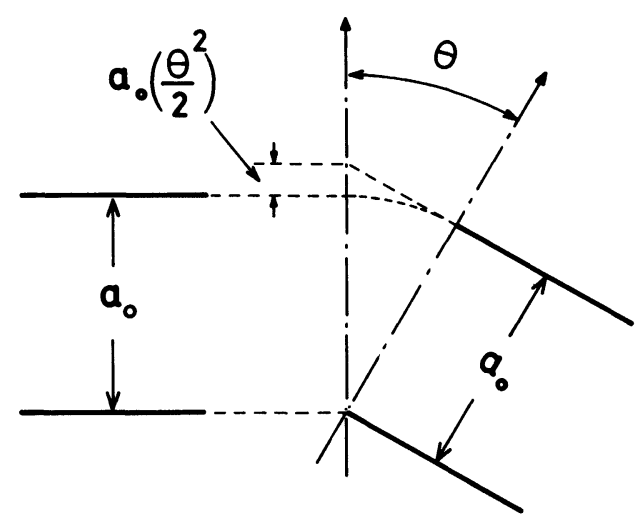

b)

FIG. 2. - a) Tilt of the molecules inside the layer. The decrease in thickness is $\simeq a_{0} \frac{\varphi_{0}^{2}}{2}$. The layer is at rest. $b$ ) Effect of a tilt of the layer. The increase of thickness measured along the $Z$ direction is $\simeq a_{0} \theta^{2} / 2$. Here the molecules remain locally normal to the layer.

compressive stress $\left({ }^{1}\right)$. To complete the description of the model, we call $K_{3}=\varepsilon K_{1}$ the Frank elastic constant associated with the bend deformation. It is introduced to take into account the effect of the solid boundaries (we assume here a molecular orientation normal to the surface). Usually $\varepsilon \simeq 1$, but it is expected to diverge [5] as $\left(T-T_{\mathrm{c}}\right)^{-v}$ (with $v=0.66$ ) close to a second order smectic-A-nematic phase transition.

The total free-energy density can now be written as :

$$
\begin{aligned}
\frac{F}{K_{1}}=\frac{1}{2} & \frac{1}{\lambda^{2}}\left[\left(X+\frac{\partial u}{\partial z}\right)-\frac{1}{2}\left(\frac{\partial u}{\partial x}\right)^{2}+\right. \\
+ & \left.\frac{1}{2} \varphi^{2}\right]^{2}+\frac{1}{2} \frac{1}{\lambda_{\perp}^{2}} \varphi^{2}+\frac{1}{2}\left[\frac{\partial}{\partial x}\left(\frac{\partial u}{\partial x}+\varphi\right)\right]^{2} \\
+ & \frac{1}{2} \varepsilon\left[\frac{\partial}{\partial z}\left(\frac{\partial u}{\partial x}+\varphi\right)\right]^{2}
\end{aligned}
$$

where $\theta$ is written as $\theta=\partial u / \partial x$.

We assume that the instability amplitude is small (i.e. $\theta$ and $\varphi$ are small compared to $X$, so that we can

( $\left.{ }^{1}\right)$ This model can also describe the behaviour of non-rigid molecules using an adjustable parameter $\alpha$ instead of the coefficient $1 / 2$ (see $\S 2.5 .2$ ). expand the first term). We can also drop the bend term, essentially because the spatial variation of $\varphi$ and $\theta$ is usually more rapid along $O x$ than along $O z$. We will later give a more accurate result which includes this term. $F$ becomes now :

$$
\begin{array}{r}
\frac{F}{K_{1}} \cong \frac{1}{2} \frac{1}{\lambda^{2}}\left[\left(X+\frac{\partial u}{\partial z}\right)^{2}-X\left(\frac{\partial u}{\partial x}\right)^{2}+X \varphi^{2}\right]+ \\
+\frac{1}{2} \frac{1}{\lambda_{\perp}^{2}} \varphi^{2}+\frac{1}{2}\left[\left(\frac{\partial^{2} u}{\partial x^{2}}+\frac{\partial \varphi}{\partial x}\right)\right]^{2}
\end{array}
$$

The instability is calculated using the following method : we assume that a periodic distortion of $\varphi$ (or $u$ ) spontaneously exists along $O x$ in a given plane at height $O z$, of the form $\varphi($ or $u)=\varphi_{0}\left(\right.$ or $\left.u_{0}\right) \cdot \exp ($ iqx $)$. This distortion could be induced for instance by the boundaries. The general expression for $\varphi(z)$ (or $u(z))$ in the bulk is derived by minimizing the free energy density $F$. For low enough values of the applied stresses $\varphi$ (or $u$ ) is found to dampen out along $z$. The instability is obtained for the stress value for which the spontaneous distortion is no more damped but is amplified in the bulk. The stress threshold is computed by supposing that the distortion of diverging amplitude is periodic along $O z$, in the form of $\varphi(z)$ (or $u(z)) \sim \varphi(x)$ (or $u(x))$. sin $\pi / d . z$ for the first mode of interest (for the higher periodic modes $\varphi(z)($ or $u(z)) \sim \varphi(x)($ or $u(x))$. sin $n \pi / d . z$, where $n$ is an integer).

2.2 TILT INSTABILITY OF THE MOLECULAR AXIS INSIDE THE LAYERS (OR BUCKLING OF THE DIRECTOR) UNDER COMPRESSIVE STRESS. - 2.2.1 Equilibrium of the molecular axis in the presence of a static deformation. - Let us assume a given periodic uniform undulation of the layers induced for instance by the boundaries, with a wave-vector $\mathbf{q}$ and of the form : $\theta(x)=\theta_{0} \exp (i q x)$. The equilibrium equation for $\varphi$ is :

$$
\varphi \cdot\left[\frac{1}{\lambda_{\perp}^{2}}+\frac{X}{\lambda^{2}}\right]-\frac{\partial^{2} \varphi}{\partial x^{2}}-\frac{\partial^{3} u}{\partial x^{3}}=0
$$

the solution of which is :

$$
\varphi=-\frac{\lambda^{2} q^{2} \theta}{X+\lambda^{2} / \lambda_{\perp}^{2}+\lambda^{2} q^{2}} .
$$

In the absence of external stress, the equilibrium molecular tilt is opposed to $\theta$, and tends to decrease the amplitude $(\theta+\varphi)$ of the angular oscillations of the director. Close to an $\mathrm{A} \rightarrow \mathrm{C}$ phase transition, $\lambda_{\perp}^{2}$ diverges and one gets essentially : $\varphi+\theta=0$, i.e. a situation corresponding to a homeotropic crystal. Far from the transition, the molecular tilt is very weak, because $\lambda^{2} q^{2}$ is much smaller than 1. Whatever its amplitude may be, the effect of the molecular tilt is to lower the Frank distortion energy.

2.2.2 Mechanical instability of the molecular axis under compressive stress. - One immediately obtains 
a molecular tilt instability (or buckling of the director) inside the layers by applying a negative stress (compression) corresponding to the strain :

$$
X=-\left(\frac{\lambda^{2}}{\lambda_{\perp}^{2}}+\lambda^{2} q^{2}\right)
$$

which induces a divergence of $\varphi$ for a finite value of $\theta$. In the limit of vanishing $q$, the compression threshold is given by :

$$
X_{\mathrm{c}}=-\lambda^{2} / \lambda_{\perp}^{2}=B_{\perp} / B
$$

We shall confirm later, by studying the coupled instability in $\varphi$ and $u$, that the molecular buckling appears first at $q=0$. For a normal smectic-A, $X_{\mathrm{c}}$ is relatively large $\left(X_{\mathrm{c}} \sim 10^{-1}\right)$ and may be difficult to realize.

However, close to an $\mathrm{A} \rightarrow \mathrm{C}$ second-order phase transition, $X_{\mathrm{c}}$ goes to zero as $\left(\frac{T-T_{\mathrm{c}}}{T_{\mathrm{c}}}\right)^{\gamma}$ and the effect should be spectacular. We can estimate how close to $T_{\mathrm{c}}$ one has to be in order to observe easily the molecular tilt. A reasonable $\left|X_{\mathrm{c}}\right|$ would be comparable to the value necessary to induce an undulation instability of layers under dilation. That occurs (see next section) for $X_{\mathrm{d}} \sim \lambda^{2} q_{\mathrm{c}}^{2}$, with $q_{\mathrm{c}}^{2}=\pi / \lambda d$. Assume a molecular field exponent value $\gamma=1 . B_{\perp}$ is of the order of $\frac{k_{\mathrm{B}} T_{\mathrm{c}}}{a b c}\left(\frac{T-T_{\mathrm{c}}}{T_{\mathrm{c}}}\right)$ where $k_{\mathrm{B}}$ is the Boltzmann constant and $a b c$ is a molecular volume. The result is $\lambda^{-2} \sim 10^{11}\left(T-T_{\mathrm{c}}\right)$ c.g.s. A $15 \mu \mathrm{m}$ thick sample gives $q_{\mathrm{c}}^{2} \sim 10^{10}$, i.e. $\left(T-T_{\mathrm{c}}\right) \sim 0.1{ }^{\circ} \mathrm{C}$ for $\left|X_{\mathrm{c}}\right| \sim X_{\mathrm{d}}$. a value which is very easy to produce. A measurement of the temperature dependence of $X_{\mathrm{c}}$ close to an A $\leftrightarrow \mathrm{C}$ transition should then give the exponent $\gamma$.

The influence of the bend term is calculated in the last section. It just adds a temperature independent term $\left(\varepsilon q_{\mathrm{c}}^{4} \lambda^{4}\right)$ to $X_{\mathrm{c}}$. This shift is very weak, even for relatively thin samples $(d=15 \mu \mathrm{m})$. With the above data one must approach as close to $T_{\mathrm{c}}$ as 30 microdegrees to observe it, and that is beyond the present experimental capabilities. This shift would be more important close to a smectic-A to nematic phase transition because of the divergence of $K_{3}$. However in that region $X_{\mathrm{c}}$ is usually too large to be produced easily and the bend-shift effect on $X_{\mathrm{c}}$ remains academic.

In practice, calling $\mathcal{F}$ the compressive force exerted by the plate on the sample of area $S$, it results directly from Hooke's law that the pressure is $\mathcal{F} / S=B \delta / d$. The tilt appears then for a critical stress that can be expressed as :

$$
\frac{\mathscr{F}_{\mathrm{c}}}{S}=B\left(\frac{\delta}{d}\right)_{\mathrm{th}}=B_{\perp}, \quad\left(\text { Since }\left(\frac{\delta}{d}\right)_{\mathrm{th}}=X_{\mathrm{c}}\right) .
$$

The modulus $B_{\perp}$ can be measured directly by the critical pressure corresponding to the tilt instability threshold. The distinction between critical stress and critical strain (previously introduced in $\S 2.2 .2$ ) may appear academic in linear elasticity of an ideal smectic.

For a real smectic, in the presence of defects, the physical quantity defining the threshold is the critical stress.

2.3 COUPLED MOLECULAR AND LAYER TILT INSTABILITIES. - The general form (2) for the free energy contains two unknown functions $u$ and $\varphi$ of $x$ and $z$. The extremum condition for $F$ gives now two coupled differential equations to describe the equilibrium situation, namely :

$$
\frac{1}{\lambda^{2}}\left(\frac{\partial^{2} u}{\partial z^{2}}-X \frac{\partial^{2} u}{\partial x^{2}}\right)-\frac{\partial^{4} u}{\partial x^{4}}=\frac{\partial^{3} \varphi}{\partial x^{3}}
$$

and one written previously :

$$
\left[\frac{1}{\lambda^{2}}+\frac{X}{\lambda^{2}}\right] \varphi-\frac{\partial^{2} \varphi}{\partial x^{2}}=\frac{\partial^{3} u}{\partial x^{3}} .
$$

We look again for periodic solutions of wave vector $\mathbf{q}$ along $O x$. We already know a situation where $\varphi$ and $u$ are decoupled ; when we make $X=X_{\mathrm{c}}$ in eq. (3) $\varphi$ becomes large, independently of $u$. Similarly, it is possible to get a solution of large $u$, independent of $\varphi$, by equating to zero the 1.h.s. of eq. (5), which gives :

$$
\frac{1}{\lambda^{2}} X q^{2}-q^{4}-\frac{\pi^{2}}{d^{2}} \frac{1}{\lambda^{2}}=0, \text { i.e. }
$$

$X=X_{\mathrm{d}} \quad$ with $\quad X_{\mathrm{d}}=\lambda^{2} q^{2}\left(1+q_{\mathrm{c}}^{4} / q^{4}\right) . \quad X_{\mathrm{d}}$ is the threshold for layer undulation under a dilative stress. $X_{\mathrm{d}}$ is minimum $\left(X_{\mathrm{d}}=2 \lambda^{2} q_{\mathrm{c}}^{2}\right)$ for $q=q_{\mathrm{c}}$ with $q_{\mathrm{c}}^{2}=\pi / \lambda d$; it corresponds to a displacement of the upper plate of $\delta=2 \pi \lambda$, independent of $d$.

Avoiding these two pathological situations, we now look at how a given undulation of $u$ and $\varphi$ along $x$ is damped inside the material, following the method of ref. [7]. We write $u \equiv v(x) \cdot w(z)$. Using (4) to eliminate $\varphi$, we obtain :

$$
\frac{\ddot{w}}{w}+\lambda^{2} q^{2} \frac{\left(X-\lambda^{2} q^{2}\right) / \lambda_{\perp}^{2}+X^{2} / \lambda^{2}}{X+\lambda^{2} / \lambda_{\perp}^{2}+\lambda^{2} q^{2}}=0
$$

2.3.1 Attenuation in the absence of strain. - For $X=0$, this equation has exponential solutions of the form $w=w_{0} \cdot \exp ( \pm z / L)$ with an attenuation length $L$ given by $L \lambda q^{2}=\left(1+\lambda_{\perp}^{2} q^{2}\right)^{1 / 2}$. Calling $L_{\mathrm{A}}$ the attenuation length in a smectic-A crystal with nontiltable molecules [7], one obtains :

$$
L=L_{\mathrm{A}}\left(1+\lambda_{\perp}^{2} q^{2}\right)^{1 / 2} .
$$

To understand this increase of $L$ in presence of tilt, we must remember that $L$ is the distance along which a spontaneous layer displacement $u$ relaxes the Frank energy implied by an initial undulation. As the molecular tilt $\varphi$ decreases the Frank energy, it takes a longer distance $L$ for $u$ to dampen out. For usual smectic-A, the difference between $L$ and $L_{\mathrm{A}}$ is negli- 
gible. It becomes important at the $\mathrm{A} \rightarrow \mathrm{C}$ transition in the same range of temperature as the one previously suggested for measuring $X_{\mathrm{c}}$. In the limit of large $\lambda_{\perp} q$. $L$ becomes essentially nematic-like for its $q$ dependence : $L=\lambda_{\perp} / \lambda .1 / q$, but with a diverging coefficient $\lambda_{\perp} / \lambda$. The nematic-like behaviour expresses the fact that there is no more coupling between the director and the layer displacement. Another interesting situation is the smectic- $\mathrm{A} \rightarrow$ nematic transition $; \lambda$ and $\lambda_{\perp}$ diverge, but their ratio is expected to remain finite. Here too $L$ has the nematic-like value $\lambda_{\perp} / \lambda .1 / q$, but it remains finite. One expects then a strong decrease of the light scattering signal from static undulations of layers induced by boundary irregularities, at the $\mathrm{A} \leftrightarrow \mathrm{N}$ transition.

2.3.2 Instabilities in the presence of strain. 2.3.2 a) General solutions. - An instability appears when, instead of damping out, a small undulation blows up along $z$. With the assumed boundary conditions, that corresponds to $\ddot{w} / w=-(\pi / d)^{2}$. (6) gives now :

$$
\begin{aligned}
X^{2}+X\left(\frac{\lambda^{2}}{\lambda_{\perp}^{2}}\right. & \left.-\frac{\pi^{2}}{d^{2} q^{2}}\right)- \\
& -\frac{\lambda^{2}}{\lambda_{\perp}^{2}}\left(q^{2} \lambda^{2}+\frac{\pi^{2}}{q^{2} d^{2}}\right)-\frac{\pi^{2} \lambda^{2}}{d^{2}}=0
\end{aligned}
$$

or, using the $q_{\mathrm{c}}$ notation :

$$
\begin{aligned}
& X^{2}+X\left(\frac{\lambda^{2}}{\lambda_{\perp}^{2}}-\frac{\lambda^{2} q_{\mathrm{c}}^{4}}{q^{2}}\right)- \\
& \quad-\frac{\lambda^{2}}{\lambda_{\perp}^{2}}\left(q^{2} \lambda^{2}+\lambda^{2} \frac{q_{\mathrm{c}}^{4}}{q^{2}}\right)-\lambda^{4} q_{\mathrm{c}}^{4}=0 .
\end{aligned}
$$

For a given $q$, there are now two threshold values $X_{+}$ and $X_{-}$for the strain giving rise to instabilities, which are the roots of $(8)$ or $\left(8^{\prime}\right)$. These roots have opposite signs, so there are in general compression and dilation instabilities which of course, need not appear exactly for $X_{\mathrm{c}}$ or $X_{\mathrm{d}}$. Physically, we are interested in the minimum value of the moduli of these roots; for a given $\lambda^{2} / \lambda_{\perp}^{2}$ (i.e. a given material at a given temperature), we look for the optimum wave-vector such that $\frac{\partial X}{\partial\left(q^{2}\right)}=0$. We obtain the extremum condition :

$$
X=\frac{\lambda^{2}}{\lambda_{\perp}^{2}}\left(\frac{q^{4}}{q_{\mathrm{c}}^{4}}-1\right)
$$

which tells us immediately that at threshold, dilation instabilities have a wave-vector equal to or larger than $q_{\mathrm{c}}$, while compression instabilities have a wavevector smaller than $q_{\mathrm{c}}$. The solutions $X_{ \pm}$are given by :

$$
\begin{aligned}
2 X_{ \pm}=-\frac{\lambda^{2}}{\lambda_{\perp}^{2}}+ & \frac{\lambda^{2} q_{\mathrm{c}}^{4}}{q^{2}} \pm\left(\frac{\lambda^{2}}{\lambda_{\perp}^{2}}+\frac{\lambda^{2} q_{\mathrm{c}}^{4}}{q^{2}}\right)^{1 / 2} \\
& \cdot\left(\frac{\lambda^{2}}{\lambda_{\perp}^{2}}+\frac{\lambda^{2} q_{\mathrm{c}}^{4}}{q^{2}}+4 q^{2} \lambda^{2}\right)^{1 / 2}
\end{aligned}
$$

Let us calculate now approximate expressions for $X_{ \pm}$ in the two limits of a rigid smectic-A $\left(\lambda_{\perp}^{2} / \lambda^{2} \rightarrow 0\right)$ and of the $\mathrm{A} \rightarrow \mathrm{C}$ transitions $\left(\lambda_{\perp}^{2} / \lambda^{2} \rightarrow \infty\right)$.

$2.3 .2 \mathrm{~b})$ The rigid smectic-A. - In the limit $\lambda_{\perp}^{2} \rightarrow 0, X_{+}$obviously tends towards $X_{\mathrm{d}} . X_{+}$is given here by $X_{+}=X_{d}-\lambda^{2} \lambda_{\perp}^{2} q^{4}$; the optimum wavevector is now $q^{2}=q_{\mathrm{c}}^{2}\left(1+\lambda_{\perp}^{2} q_{\mathrm{c}}^{2}\right)$. These expressions apply very well for a normal smectic-A, where $\lambda_{\perp} q_{\mathrm{c}} \ll 1$. The decrease in threshold when molecular tilt is allowed is easy to understand : the undulation appears when it is energetically more favourable to produce a Frank distortion than a uniform dilation. As the Frank energy is relaxed by the molecular tilt, the threshold is lower,$\varphi$ remains very small compared to $\theta$.

To calculate $X_{-}$, we assume that not only $\lambda_{\perp}$ but also $q$ goes down to zero. The diverging terms $\lambda^{2} q_{\mathrm{c}}^{4} / q^{2}$ cancel exactly and one finds $X_{-}=-\lambda^{2} / \lambda_{\perp}^{2}=X_{\mathrm{c}}$, with $q=0$, from the extremum condition (9). $\varphi$ diverges above $X_{\mathrm{c}}$ and is essentially uncoupled to $u$.

$2.3 .2 \mathrm{c}$ ) The $\mathrm{A} \rightarrow \mathrm{C}$ transition (non-rigid smectic-A). - When $\lambda^{2} / \lambda^{2}$ diverges, $X_{+}$is given by :

$$
2 X_{+}=\lambda^{2} \frac{q_{\mathrm{c}}^{4}}{q^{2}}\left[1+\left(1+4 \frac{q^{4}}{q_{\mathrm{c}}^{4}}\right)^{1 / 2}\right]
$$

a finite optimum value for $q,\left(q>q_{\mathrm{c}}\right)$, is impossible because the extremum condition (9) would give $X \rightarrow 0$, incompatible with (11); $q$ should then diverge, and $X_{+}$is given by :

$$
X_{+}=\lambda^{2} q_{\mathrm{c}}^{2}=\frac{1}{2} X_{\mathrm{d}} .
$$

One gets the optimum $q$ from (9) : $q^{2}=q_{\mathrm{c}}^{2} \lambda_{\perp} q_{\mathrm{c}}$, which diverges. From (4), we note that $(\theta+\varphi)$ is zero. One can visualize this undulation instability of infinite $q$ as a smectic-A to a quasi-nematic phase transition under dilation, since molecules slide against each other to counteract the applied strain. This picture is of course of limited interest, since non-linear terms in the expression of the energy density $F$ become important for large $\theta$ or large $\varphi$. The second threshold $X_{-}$is now given by

$$
X_{-}=-\frac{\lambda^{2}}{\lambda_{\perp}^{2}}-\lambda^{2} q^{2}
$$

the optimum $q$ is obviously $q=0$, and $X_{-}=X_{\mathrm{c}}$. This result is in fact of a more general validity. From the optimum relationship (9) one deduces that $X_{-}$can vary from 0 to $X_{\mathrm{c}}$, when $q$ goes from $q_{\mathrm{c}}$ to zero. Now, substituting $X=X_{\mathrm{c}}$ in (8), we find that $X_{\mathrm{c}}$ is always included between $X_{+}$and $X_{-}$. The compressional minimum threshold is then always $X_{-}=X_{\mathrm{c}}$ with $q=0 ; \varphi$ diverges independently of $u$.

One can now draw a diagram giving the evolution of $X_{+}$and $X_{-}=X_{\mathrm{c}}$ versus $\lambda_{\perp}^{2} / \lambda^{2}$ (Fig. 3). This diagram allows us to predict that close to the $\mathrm{A} \rightarrow \mathrm{N}$ phase transition, because the ratio $\lambda_{\perp}^{2} / \lambda^{2}$ is constant, the 
measurement of $X_{+}$is a good way to follow the divergence of $\lambda$, the corrective factor coming from the possible molecular tilt remaining constant.

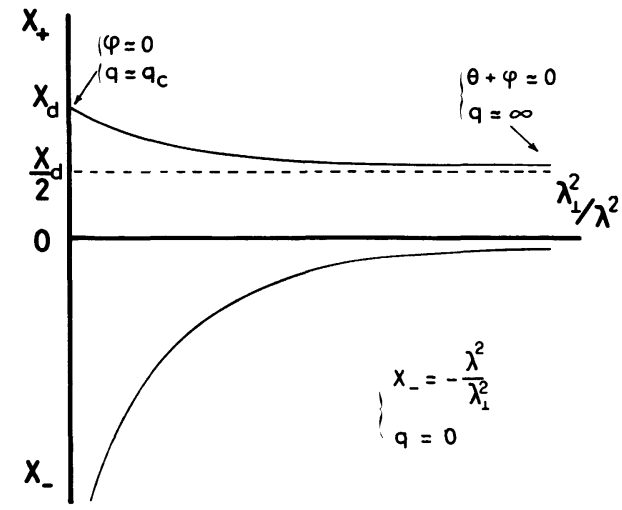

Fig. 3. - Diagram of the evolution of the thresholds $X_{+}$for the compression instability and $X_{-}$for the dilation instability, as a function of the parameter $\lambda_{\perp} / \lambda$ characteristic of the smectic phase.

2.4 INFLUENCE OF THE BEND OF THE DIRECTOR. $u$ and $\varphi$ are now integrals of the equations :

$$
\begin{aligned}
-\frac{X}{\lambda^{2}} \frac{\partial^{2} u}{\partial x^{2}}+\frac{1}{\lambda^{2}} & \frac{\partial^{2} u}{\partial z^{2}}-\frac{\partial^{4} u}{\partial x^{4}}- \\
& -\varepsilon \frac{\partial^{4} u}{\partial x^{2} \partial z^{2}}=\frac{\partial^{3} \varphi}{\partial x^{3}}+\varepsilon \frac{\partial^{3} \varphi}{\partial x \partial z^{2}}
\end{aligned}
$$

and

$$
\left(\frac{1}{\lambda_{\perp}^{2}}+\frac{X}{\lambda^{2}}\right) \varphi-\frac{\partial^{2} \varphi}{\partial x^{2}}-\varepsilon \frac{\partial^{2} \varphi}{\partial z^{2}}=\frac{\partial^{3} u}{\partial x^{3}}+\varepsilon \frac{\partial^{3} u}{\partial x \partial z^{2}}
$$

For a periodic solution of wave-vector $\mathbf{q}$, one gets the compatibility relationship :

$$
\begin{aligned}
{\left[X-X_{\mathrm{d}}-\varepsilon q_{\mathrm{c}}^{4} \lambda^{4}\right]\left[X-X_{\mathrm{c}}\right.} & \left.+\varepsilon q_{\mathrm{c}}^{4} \lambda^{4}\right]+ \\
& +\left[q^{2} \lambda^{2}+\varepsilon q_{\mathrm{c}}^{4} \lambda^{4}\right]^{2}=0
\end{aligned}
$$

and one immediately sees that the new thresholds $X_{\mathrm{d}}$ and $X_{\mathrm{c}}$ are shifted upwards by the quantity $X_{\mathrm{s}}=\varepsilon q_{\mathrm{c}}^{4} \lambda^{4}$. This shift simply represents the additional dilation or compression necessary to balance the bend energy; in effect,

$$
\frac{1}{\lambda^{2}} X_{\mathrm{s}} \varphi^{2}=\varepsilon \frac{\pi^{2}}{d^{2}} \varphi^{2}
$$

gives $X_{\mathrm{s}}=\varepsilon q_{\mathrm{c}}^{4} \lambda^{4}$. In practice $X_{\mathrm{s}}$ is hardly visible, even when $\varepsilon$ diverges (for instance close to a smectic-Anematic second-order transition). The new extremum condition is here :

$$
X=\frac{\lambda^{2}}{\lambda_{\perp}^{2}}\left(\frac{q^{4}}{q_{\mathrm{c}}^{4}}-1\right)-\varepsilon q_{\mathrm{c}}^{4} \lambda^{4}
$$

and the new displacement threshold in dilation is :

$$
\delta=2 \pi \lambda\left(1+\frac{\varepsilon}{2} q_{\mathrm{c}}^{2} \lambda^{2}\right)=2 \pi \lambda\left(1+\frac{\pi}{2} \frac{\varepsilon \lambda}{d}\right) ;
$$

the correction factor diverges as $\left(T-T_{\mathrm{c}}\right)^{-1}$ since

$$
\varepsilon=\frac{K_{3}}{K_{1}} \sim\left(\frac{T_{\mathrm{c}}-T}{T_{\mathrm{c}}}\right)^{-0.66}
$$

and

$$
\lambda \sim\left(\frac{T_{\mathrm{c}}-T}{T_{\mathrm{c}}}\right)^{-0.33}
$$

(see ref. [6, 26]). Starting from a value of the order of $3 \times 10^{-4}$ for the correction factor [taking the worst case : $d \sim 30 \mu \mathrm{m}$ and $\varepsilon \sim 2$ ] at a few degrees off $T_{\mathrm{c}}$, one has to go as close as a few millidegrees from $T_{\mathrm{c}}$ to see its effects.

When $\lambda_{\perp}^{2}$ diverges, a simple calculation shows that $X_{+}$is not affected at all by the bend. since the Frank energy is totally relaxed by the molecular tilt.

2.5 Amplitude OF THE DEFORMations ABOVE THRESHOLD. - For simplicity, the amplitudes $u_{0}$ and $\varphi_{0}$ are calculated separately in the two simple cases of pure undulation of layers under dilation $\left(\rightarrow u_{0}\right)$, and pure molecular tilt under compression $\left(\rightarrow \varphi_{0}\right)$. To do so, we take in the linear approximation (close to threshold) solutions in the form of periodic functions of the appropriate variables. The free-energy density $F$ is integrated over the sample volume to eliminate the spatial variation. The odd powers of the amplitude $\left(u_{0}\right.$ or $\left.\varphi_{0}\right)$ vanish and $F$ can be expressed as a polynomial in $u_{0}^{2}$ (or $\varphi_{0}^{2}$ ) which is a kind of Landau expansion versus the order parameter $u$ (or $\varphi$ ). We only keep the two first powers in this expansion. The equilibrium amplitude corresponds to

$$
\frac{\partial F}{\partial\left(u_{0}^{2}\right)} \equiv 0, \quad \text { and } \quad \frac{\partial F}{\partial\left(\varphi_{0}^{2}\right)} \equiv 0
$$

in each case.

2.5.1 Undulation instability of the layers. - We use for $F$ the simplified form in which $q=q_{\mathrm{c}}$ :

$$
\frac{F}{K_{1}}=\frac{1}{2 \lambda^{2}}\left[X+\frac{\partial u}{\partial z}-\frac{1}{2} \theta^{2}+\frac{1}{24} \theta^{4}\right]^{2}+\frac{1}{2} q_{\mathrm{c}}^{2} \theta^{2}
$$

where $\theta=\partial u / \partial x, X=\delta / d$, and $\delta$ is the imposed displacement.

In the bracket we keep the $\left\{\theta^{4}\right\}$ term coming from the expansion of $\cos \theta$. The undulation amplitude is assumed to be in the form :

$$
u=u_{0}\{\cos (\pi / d) \cdot z\} \cdot \sin q_{\mathrm{c}} x
$$


One gets after integration over $x$ from 0 to $2 \pi / q_{\mathrm{c}}$ and over $z$ from $-d / 2$ to $d / 2$ :

$$
\begin{aligned}
\int_{0}^{2 \pi / q_{\mathrm{c}}} \mathrm{d} x & \cdot \int_{-d / 2}^{d / 2} \mathrm{~d} z \cdot F / K_{1}=\left(\frac{\pi}{4 \lambda^{2}}\right) \cdot \\
& \cdot q_{\mathrm{c}} u_{0}^{2} \cdot\left(\delta_{\mathrm{th}}-\delta\right)+\frac{3}{256}\left(\frac{\delta}{d}+3\right) \pi d q_{\mathrm{c}}^{3} \cdot u_{0}^{4} .
\end{aligned}
$$

Above the threshold defined by $\delta_{\text {th }}=2 \pi \lambda$ one obtains for the amplitude, after making $\delta / d \ll 1$,

$$
u_{0}=\frac{8}{3} \lambda\left[\frac{\delta-\delta_{\mathrm{th}}}{\delta_{\mathrm{th}}}\right]^{1 / 2} .
$$

The linear regime is defined by $\theta \ll 1$, or $u_{0} \ll(\lambda d / \pi)^{1 / 2}$. Experimentally, as will be exposed later we need to explore, at most, a range of displacements $\delta$ such that $\delta / \delta_{\text {th }}<2$ in order to determine the threshold value $\delta_{\text {th }}$. For $\delta=2 \delta_{\text {th }}$ we would get $u_{0} \simeq 90 \AA$ and $\theta \simeq 10^{-2} \mathrm{rad}$. Thus the condition for the linear regime is always fulfilled.

2.5.2 Tilt instability of the molecules inside the layers. - We use the same procedure with :

$$
\begin{aligned}
\frac{F}{K_{1}}=\frac{1}{2 \lambda^{2}}[( & \left.\left.X+\frac{\partial u}{\partial z}\right)-\frac{1}{2}\left(\frac{\partial u}{\partial z}\right)^{2}+\frac{1}{2} \varphi^{2}\right]^{2}+ \\
& +\frac{1}{2 \lambda_{\perp}^{2}} \varphi^{2}+\frac{1}{2}\left[\frac{\partial}{\partial x}\left(\frac{\partial u}{\partial x}+\varphi\right)\right]^{2}+ \\
& +\frac{1}{2} \varepsilon\left[\frac{\partial}{\partial z}\left(\frac{\partial u}{\partial x}+\varphi\right)\right]^{2}+\frac{1}{4} \frac{C}{K_{1}} \varphi^{4}
\end{aligned}
$$

and we assume a solution of the form $\varphi=\varphi_{0} \cos (\pi / d) z$. Starting from a perfect unperturbed sample (i.e. neglecting the case of an undulated structure due to static deformations), we only have to compute the integral of $F$ over the thickness $\{-d / 2 \rightarrow d / 2\}$; we obtain the expression :

$$
\begin{array}{r}
\int_{-d / 2}^{d / 2} \mathrm{~d} z \cdot F / K_{1}=\frac{d}{4}\left[\frac{X}{\lambda^{2}}+\frac{1}{\lambda_{\perp}^{2}}+\varepsilon \frac{\pi^{2}}{d^{2}}\right] \varphi_{0}^{2}+ \\
+\frac{3}{32} d\left(\frac{1}{2 \lambda^{2}}+\frac{C}{K_{1}}\right) \varphi_{0}^{4},
\end{array}
$$

and the tilt amplitude above threshold is now :

$$
\varphi_{0}=\frac{2}{\sqrt{3}}\left(\frac{\lambda}{\lambda_{\perp}}\right)\left[\frac{1+\left(\varepsilon \pi^{2} \lambda_{\perp}^{2} / d^{2}\right)}{1 / 2+C \lambda^{2} / K_{1}}\right]^{1 / 2}\left|\frac{X-X_{\mathrm{c}}}{X_{\mathrm{c}}}\right|^{1 / 2} .
$$

Here we see that, to remain in the linear regime $\left(\varphi_{0} \ll 1\right)$, one needs to use very low relative strains. In effect $\left|X-X_{\mathrm{c}} / X_{\mathrm{c}}\right|$ must be less than about $3 \times 10^{-2}$ for $\varphi_{0}<10^{-2} \mathrm{rad}$.

From these results we expect to find a linear variation of the square of the deformation amplitude versus the applied strain, for both instabilities. As will be shown later this is observed in a light scattering experiment, provided the scattering geometry is chosen in such a way that the scattered light intensity is proportional to $u_{0}^{2}\left(\operatorname{or} \varphi_{0}^{2}\right)$.

Remark : In the simple model described here we made the assumption of rigid rod-like molecules. Taking into account the possibility of the molecule bending would lead to the introduction of new terms in $\theta^{2}$ and $\theta^{4}$ (or $\varphi^{2}$ and $\varphi^{4}$ ) in the above expressions. In effect the molecules are generally composed of two parts : a central part made of aromatic nuclei, which is very rigid, and the aliphatic chain which may be flexible. Moreover the flexibility of this chain may vary with temperature $[8,9]$ and under mechanical stresses. Thus in a more refined model the elastic description of the layers should include at least these terms. For example one could introduce in the first bracket of the free-energy density an adjustable parameter $\alpha$ before the $\varphi_{0}^{2}$ term.

2.6 SiMPLE MODEL TO DESCRIBE THE DYNAMICS OF THE INSTABILITIES. - The elastic behaviour of the structure under stresses normal to the layers is strongly dependent on the physical characteristics of the crystal. In order to describe in the simpler way the dynamics of the instabilities we must take into account :

- the motion of structural defects still present in a real sample,

- the hydrodynamical flow,

- the influence of the boundaries on the velocity field.

2.6.1 Motion of elementary defects of structure. The simplest defects we may think of are edge dislocations (Fig. 4a) that can move under the action of the external forces applied to induce the instabilities. These dislocations glide between the layers in order to compensate the effect of the uniaxial stress. This motion ( climb) is controlled by the permeation process. Such a motion has the effect of adding or subtracting extra layers when respectively dilative or compressive forces are applied, and tends to relax the applied stress. Therefore, it should control the dynamics and the duration of the instability.

2.6.1 a) Simple model for the calculation of the dislocation velocity. - Let us consider an edge dislocation of Burgers vector $b$ contained in the middle plane of a perfect sample (Fig. $4 b$ ). At equilibrium the compression energies per elementary volume defined as : $d . L . k$ (where $d$ is the sample thickness, $L$ is the edge-dislocation line length and $k$ is some arbitrary length unit along the $x$ direction) can be written as :

$$
E_{1}=\frac{1}{2} B\left(\frac{\delta+b / 2}{d}\right)^{2} \cdot d \cdot L \cdot k, \quad \text { in region } 1,
$$

and

$$
E_{2}=\frac{1}{2} B\left(\frac{\delta-b / 2}{d}\right)^{2} \cdot d \cdot L \cdot k, \quad \text { in region } 2
$$



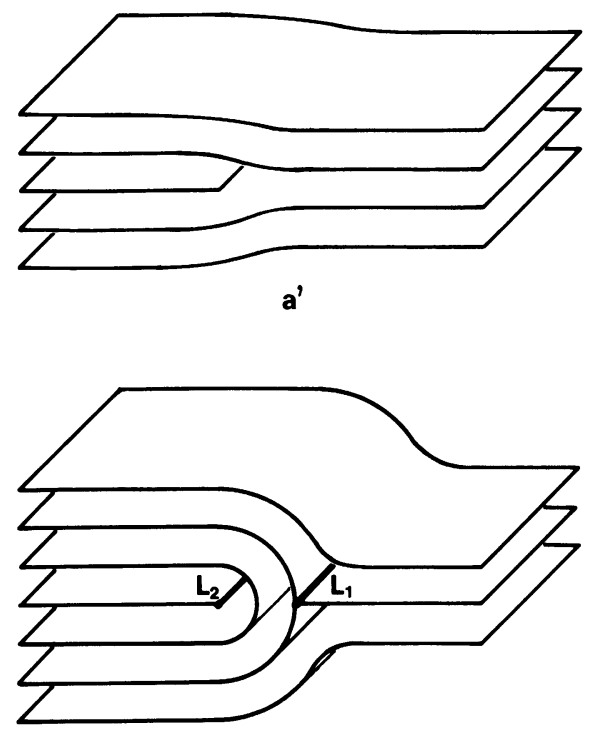

b'

a)

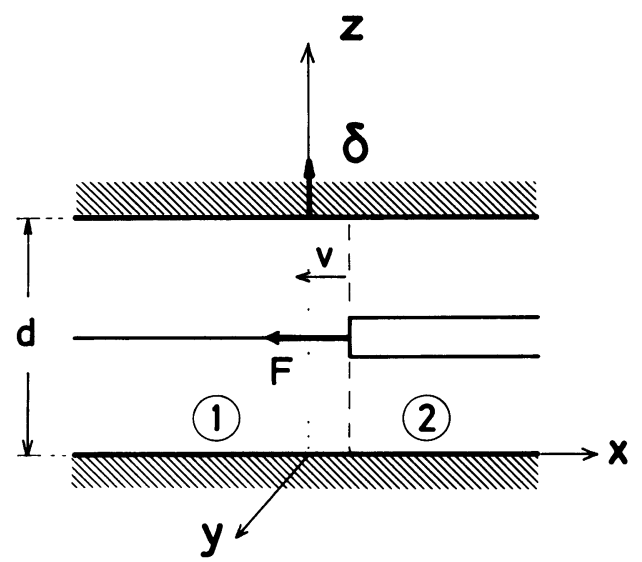

b)

Fig. 4. - a) Two types of edge dislocations. The larger one sketched here is in fact composed of two disclinations of opposite sign. $b$ ) Motion of an elementary edge dislocation under a dilative force. (The dilation is $\delta$ ). $F$ is the friction force and $v$ is the dislocation velocity. c) Periodic distribution of the strains inside a smectic

The energy variation in a displacement value $x$ of the dislocation is then :

$$
\Delta E=B \frac{L}{d} \cdot \delta \cdot b \cdot x,
$$

and the friction force per line of unit length is :

$$
F=\frac{1}{L} \frac{\partial E}{\partial x}=\frac{B}{d} \cdot \delta \cdot b .
$$

Equating this expression to the one given by de Gennes [25] for the friction force as :

$F=\eta \kappa b V$ (where $\eta$ is a viscosity and $\kappa$ is a parameter of the order $\mathrm{a}^{-1}$ ), we get for the velocity $V$ the expression :

$$
V \simeq \frac{B a \delta}{d \eta}
$$

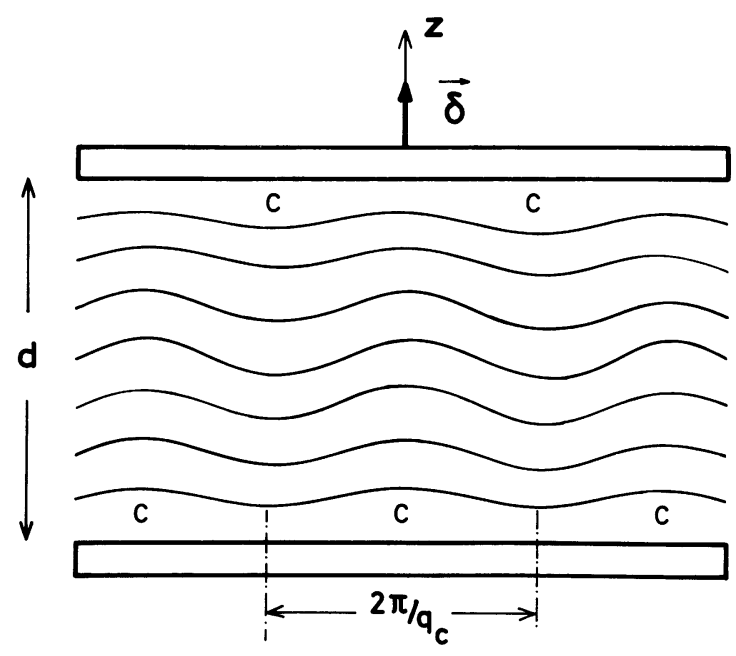

c)

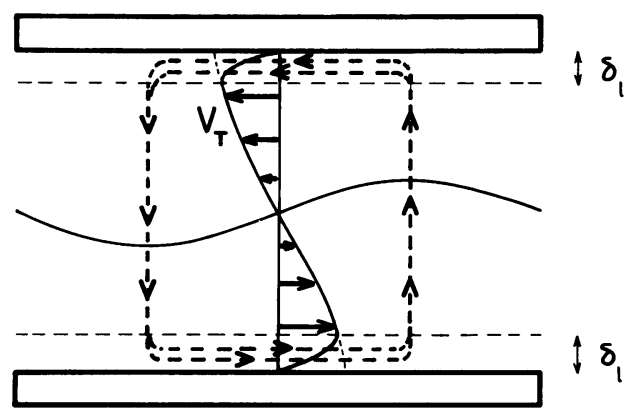

d)

crystal subjected to a dilative force. The regions $\mathrm{C}$ are undergoing the highest strains. d) Approximate model for the distribution of the velocities in an undulated structure. The tangential velocity $V_{T}$ vanishes over a thickness $\delta_{l}$ (boundary layer) of the order of some molecular lengths.

2.6.1 b) Transit times of the dislocations : The dislocations which contribute to the dynamics of the instabilities can be supposed to have different localizations or origins.

First a finite density of dislocations of opposite sign can exists everywhere in the bulk,

- some dislocations can be nucleated from the surface irregularities or from anchoring defects ;

- others can be attracted towards regions of high strains resulting in the local stress relaxation. In a perfectly undulated structure where the undulation amplitude vanishes on the solid boundaries such regions are periodically located close to these boundaries (Fig. $4 c$ ), the spatial period being equal to $2 \pi / q_{\mathrm{c}}$ at threshold. However in the middle of the sample thickness the normal strain is constant ; 
- in the bulk, but far from the central part, we may suppose a constant density of dislocations per unit area. When undulations occur these dislocations are trapped inside the regions of high deformation. At threshold the distance between these regions is of about $\pi / q_{\mathrm{c}}$.

One can also imagine that edge dislocations come from the edges of the sample and relax the total stress on the whole sample area. They are expected to move in the central part where the stress is constant along $O z$.

In any case the applied stress is totally relaxed when the number $n$ of extra layers coming into the studied area is at least such that $n . a=2 \pi \lambda$ i.e. $n \simeq 6$ (since $\lambda \simeq a$ ).

The characteristic relaxation times for these processes are given by the transit times of the defects. The time corresponding to the first process is

$$
\tau_{1} \simeq \frac{\pi}{q_{\mathrm{c}} V} .
$$

Taking the values $B \simeq 2 \times 10^{7} \mathrm{cgs}$ (ref. [1]), $\eta=0.4 \mathrm{cgs}$. $\kappa^{-1} \simeq 20 \AA$ and $d=100 \mu \mathrm{m}$ we get $\tau_{1} \simeq 50 \mathrm{~m}$.s for the relaxation time of the local stress. For dislocations coming from the bulk the transit time through the observed area (area illuminated by the laser beam of radius $r \simeq 0.5 \mathrm{~mm}$ ) we get a typical time $\tau_{2} \simeq r / V \simeq 25 \mathrm{~s}$, for the uniform relaxation of the stress. In fact the typical transit times should be smaller in a sample with large layer defects. In that case the distance to take into account is likely to be smaller than the laser beam radius $r$.

For comparable dislocation densities we expect the transit times (over the same distances) to be much shorter when compressive forces are applied. because close to threshold the relative displacement $\delta / d$ is much larger than when a dilative stress is applied. Then the relaxation of the stress is expected to occur within a much shorter time. In effect, $\delta / d$ is typically ten times larger in the compression experiment than in the dilation one.

2.6.1 c) Temperature dependence of these relaxation times close to second-order phase transitions. Dilative stress : Using simple arguments from Brochard [26] and de Gennes [5] it is possible to show with the expression found for the dislocation velocity. that close to a second order smectic-A to nematic transition this velocity should increase and the relaxation time $\tau_{\mathbf{d}}$ decrease. In effect $\mathbf{B}$ varies as $\left(T_{\mathrm{c}}-T\right)^{0.66}, \kappa \sim\left(T_{\mathrm{c}}-T\right)^{1 / 2}$ and $\tau_{\mathrm{d}}$ should vary like $\left(T_{\mathrm{c}}-T\right)^{-0.16}$ assuming a constant Burgers vector. Thus in our model the temperature dependence of the relaxation time is not expected to be very important.

- Compressive stress : Close to a second-order smectic-A to smectic-C phase transition the elastic modulus $B$ is not expected to show a temperature dependence. The viscosity $\eta$ is expected to show a negligible variation [26]. However, close to a smec-
tic-C phase our model for the dislocation velocity may be quite inadequate. It is suggested by $\mathrm{F}$. Brochard (private communication) that in effect the dislocation motion would be limited by the slower sound wave. Here it would be the second sound (fluctuations of layer thickness with a constant density) that controls the edge-dislocation motion. And since close to an $\mathrm{A} \rightarrow \mathrm{C}$ transition the second sound velocity decreases [26], a corresponding decrease in the dislocation velocity (and thus an increase of the relaxation time of the instability) could be expected.

2.6.2 The hydrodynamic flow; boundary layer. De Gennes has calculated [25] the thickness variation rate of a defect-free smectic-A sample undergoing stresses normal to the layers. He introduces the concept of a boundary layer in order to satisfy the boundary conditions for the tangential velocity field and for the pressure. This boundary layer $\delta_{1}$ is expressed as $\delta_{1}^{2} \sim x \cdot \kappa^{-1}$ where $x$ is the distance measured along the boundaries over which the velocity (or pressure) gradient occurs (Fig. $4 d$ ). $\kappa^{-1}$ is of order of a molecular length. When a force $F$ is exerted normal to the layers of a smectic sample the thickness variation rate $d(t)$ is given as :

$$
d(t)=d(0)\left\{1+\left|\frac{F \cdot t}{\eta R^{5 / 2} \cdot \kappa^{3 / 2} \cdot d(0)}\right|^{-4}\right\}
$$

where $d(0)$ is the initial sample thickness. $R$ the sample radius $; \kappa^{-1}$ is the parameter previously mentioned, which is defined as : $\kappa^{-1}=\left(\lambda_{\mathrm{p}} \eta\right)^{1 / 2}, \lambda_{\mathrm{p}}$ being the permeation coefficient. It is the permeation process which controls the displacement velocity of the plates as long as $\delta_{1}$ has a value much smaller than the sample thickness. When $\delta_{1}$ is comparable to, or larger than $d$, the hydrodynamic flow is described by the classical Poiseuille law. In our system both situations could be met since we use large thicknesses for the dilation experiment $(100 \mu \mathrm{m}$ to $800 \mu \mathrm{m})$ and very small ones for the compression experiment (down to $6 \mu \mathrm{m}$ ).

Now, in order to achieve instabilities we must produce a displacement $\delta$ at least equal to $\delta_{\text {th }}$ within a time $\tau$ smaller than the rise-time $\tau_{\mathrm{r}}$ of these instabilities. In addition this value must always be smaller than the time $\tau_{\mathrm{f}}$ needed by the flow to fill (or to empty according to the sign of the applied stress) the sample volume.

As an example, for the undulation experiment where typically $R=1 \mathrm{~cm}, \eta \simeq 0.1 \mathrm{cgs}, d(0) \simeq 100 \mu \mathrm{m}$, $d(t)-d(0)=\delta_{\text {th }} \simeq 120 \AA$ and $F \simeq 10^{4}$ dynes, we find that the hydrodynamic flow time $\tau_{\mathrm{f}}$ is about $10^{-1} \mathrm{~s}$. For a typical compression experiment where the force exerted is about $2 \times 10^{5}$ dynes this time would be of order of some $10^{-2} \mathrm{~s}$. In any case these times are to be compared with the rise time of the instabilities in order to know whether the instability has enough time to settle. This point is discussed in the next section.

2.6.3 Rise-time of the instabilities. - In the absence of any hydrodynamic flow, and in a perfect sample 
(defect-free) we can give an estimate of the time $\tau_{\mathrm{r}}$ needed for the establishment of the instabilities when step-like stresses are applied.

For the undulation instability [1] :

$$
\begin{aligned}
& \tau_{\mathrm{r}} \simeq \frac{\eta}{B \cdot \delta / d} \\
&\left(\simeq 10^{-4} \text { s. close to threshold }\right)
\end{aligned}
$$

and for the tilt instability :

$$
\tau_{\mathrm{r}} \simeq \frac{\eta}{B \varphi}
$$

( $\simeq 10^{-4}$ s. above threshold where $\left.\varphi \simeq 10^{-2} \mathrm{rad}.\right)$

We see that this rise-time is, in typical situations. much shorter than the hydrodynamic time and the relaxation time due to the dislocation motion. Therefore even if the flow and the dislocation motion control the lifetime of the instabilities produced they should not prevent the instabilities from being set up.

2.6.4 The two dimensional grating. - In the preceding theory the deformation of the layers under dilative stress occurs in one dimension. Therefore we expect undulations with only one wave-vector and the texture predicted is a simple grating. However Delrieu [27] has calculated that above a second threshold higher than the first one a double crossed grating could appear with two wave-vector $\mathbf{q}$ and $\mathbf{q}^{\prime}$ of equal length, and the free-energy minimum is obtained when they are orthogonal in the plane of the layers.

2.6.5 Remarks. - The expression for the thickness variation rate includes the parameter $\kappa$, which is temperature dependent. Close to a second-order smectic-A to nematic transition $\kappa$ is expected to vary as $\left(T-T_{\mathrm{c}}\right)^{1 / 2}$, provided $\eta$ does not show any temperature dependence. In that case the boundary layer should vary as $\left(T-T_{\mathrm{c}}\right)^{-1 / 4}$, and then the hydrodynamic time would also vary. Nevertheless as we use relatively thick samples close to the $\mathrm{A} \rightarrow \mathrm{N}$ transition, this variation is too small and cannot be detected. It might be detected close to a second-order $\mathrm{A} \rightarrow \mathrm{C}$ transition (where we use thin samples), but then the temperature dependence of the parameters is unknown (the permeation process seems to be a little more complicated).

Furthermore, close to an $\mathrm{A} \leftrightarrow \mathrm{C}$ transition we might expect some new hydrodynamic deformation of structure under stresses due to the coupling between the molecular orientation and a hydrodynamic flow induced for example when the sample plates are not exactly parallel. This coupling could lead to some new structural instability. As we will see later, we have in fact observed a periodic deformation that could be interpreted in such a way.
3. Experimental results. - 3.1 EXPERIMENTAL METHODS. - A homeotropic smectic-A liquid crystal is optically uniaxial, with its optical axis parallel to the common direction of the molecules (and therefore normal to the limiting surfaces). Deformations of the structure strongly modulate the dielectric anisotropy tensor of the liquid crystal, and therefore can be detected and analyzed using a light-scattering technique. The scattering wave-vector associated with the molecular tilt instability is expected to be $q \simeq 0$, while it should have a well-defined value $q_{\mathrm{c}}$ for the undulation instability of layers (with $\mathbf{q}_{\mathbf{c}}$ parallel to the plane of the layers) and the geometry needed for the observation of these instabilities will be somewhat different for each experiment.

3.1.1 Optical conditions for observation of the undulation instability. - Scattering geometry. - The mode of undulation of the layers is equivalent to a splay mode of director (assuming that the molecules are, locally, remaining normal to the layers); the wave-vector $q$ of the undulation lies in a plane parallel to the layers (i.e. normal to the optical axis). The angular distribution of the scattered intensity is found [11] by using the selection rules defined for scattering by a splay mode in nematics [10]; the new feature is that we now have to take into account the uniaxial character of the crystal. Then an incident light beam tilted away from the normal and ordinarily polarized (of wave-vector $\mathbf{k}_{\mathbf{0}}$ ) will give rise to scattered light extraordinarily polarized (and vice-versa). We call $\mathbf{k}_{\mathrm{e}}^{\prime}$ its associated wave-vector. We can represent geometrically the momentum conservation law of the scattering : the undulation wave-vector $\mathbf{q}$ will transfer the extremity of the wave-vector $\mathbf{k}_{0}$ from a sphere (wave-vector surface for the ordinary index of refraction) to an ellipsoid (surface for the extraordinary index) (Ref. to Fig. 8a). With the constraint that $\mathbf{q}$ remains in a plane normal to the optical axis one sees that the locus of the extremity of $\mathbf{k}_{\mathrm{e}}^{\prime}$ (in the process $\mathbf{k}_{0}-\mathbf{k}_{\mathrm{e}}^{\prime}$ ) is a circle defined by the intersection of the ellipsoid with the plane containing q. In the case of the process $\mathbf{k}_{\mathrm{e}} \rightarrow \mathbf{k}_{\mathrm{e}}^{\prime}$ the circle is in the same plane and inside the previous one for a positive crystal. Then the scattering occurs in a general case on two co-axial cones centered on the optical axis (Ref. to Fig. 8b). However, there is no scattering along the observation directions defined by the intersection of these cones with the incidence plane. An observation screen is placed parallel to the sample and the scattering appears on two concentric circles as it is observed for static undulations due to defects [11] with which a large number of wave-vectors is associated. When scattering occurs on the proper circle, one can relate the scattering wave-vector to the distance on the screen, between the laser spot and the observed point on the circle [12]. Now if the undulation due to the instability is uniform the corresponding wave-vector $\mathbf{q}_{c}$ will be unique in direction. The probability of finding its extremity on the scattering circle is very low. In that 
case the scattering condition can be fulfilled by rotating the sample in its plane around the optical axis. If the scattering volume is composed of many domains with arbitrary directions for $\mathbf{q}_{\mathbf{c}}$, the extremity of the wavevectors $\mathbf{q}_{\mathrm{c}}$ can be expected to be randomly distributed on a circle centered on the beam spot. Then. scattering should always be visible as two bright spots, at the intersections of this circle with the scattering circle corresponding to the chosen incoming polarization. As will be shown later, it is necessary in our experiment to rotate the sample frequently mainly because the distribution on the circle of radius $q_{\mathrm{c}}$ shows a strong maximum in an arbitrary but reproducible direction for a given sample. The optical signal due to the diffraction by the undulation is then analyzed by a phototransistor looking at the previously defined spots. Calling $\theta$ the deformation angle, it results from dipolar radiation theory that the scattered intensity at an angle $\psi$ (with respect to the molecular axis) which is large compared to $\theta$, is $I \sim \theta^{2}$. And since above threshold we have $\theta^{2} \sim\left(\delta-\delta_{\mathrm{th}} / \delta_{\mathrm{th}}\right)$, the scattered intensity $I$ is expected to be linear in displacement : $I \sim\left(\delta-\delta_{\mathrm{th}} / \delta_{\mathrm{th}}\right)$.

- Measurement of the wave-vector $q_{\mathrm{c}}$. The method used to determine the scattering wave-vector $\mathbf{q}_{\mathrm{c}}$ is the following one : on the screen parallel to the plane of the layers one measures the angle $\rho$ between the traces of the incidence plane and the scattering plane on the observation screen. The modulus of the scattering wave-vector $\mathbf{q}$ is given by the expression :

$q=\left(\frac{2 \pi}{\lambda_{0}}\right)^{2} \cdot \sin ^{2} \cdot \alpha\left\{1+\left(\frac{n_{0}}{n_{\mathrm{e}}}\right)^{2}-2 \frac{n_{0}}{n_{\mathrm{e}}} \cdot \cos \rho\right\}$.

where $\alpha$ is the incidence angle. $\lambda_{0}$ the incident light wave-length, $n_{0}$ and $n_{\mathrm{e}}$ the main refractive indices of the crystal. We have also used an observation screen normal to the transmitted laser beam at a distance $d$ from the sample. This geometry allows us to determine $q$ by the measurement of the distance $x_{\mathrm{s}}$ between the laser trace and the observation point $s$ on the scattering pattern which is now an ellipse. through a more complicated relationship [12]. However for small wave-vectors $q$ ( $q$ at least two times smaller than the light wave-vector) this relationship can be approximated by the following one :

$$
q\left(\mathrm{~cm}^{-1}\right) \simeq\left(\frac{2 \pi}{\lambda_{0}}\right) \frac{x_{\mathrm{s}}}{d},
$$

making possible rapid measurements with an error in the wave-vector less than $5 \%$.

Note : Close to a second-order smectic-A nematic phase transition. $q_{\mathrm{c}}$ is expected to vanish. as previously explained. This variation forces us to readjust the detector alignment on the spots.

3.1.2 Optical conditions for observation of the molecular tilt instability. - This instability produces a uniform deformation within each layer, and appears with a zero wave-vector (excepted in the optical axis direction where $q_{\mathrm{z}}=\pi / d$, with $d$ sample thickness). So the optical observation is made on the transmitted beam. The sample is placed between crossed linear polarizers. In an unperturbed sample the transmitted light intensity is close to zero. When a compressive stress exceeding the threshold value is applied, a part of the incident light is transmitted through the analyzer. If the incident beam were parallel to the optical axis, the phase difference between ordinary and extraordinary components would increase as $\varphi^{2}$, the intensity of light scattered in the same direction by the tilting $\varphi$ would be $I \sim \varphi^{4}$, while for an incident beam tilted away from the optical axis this intensity is in general $I \sim \varphi^{2}\left(X-X_{\mathrm{c}}\right) / X_{\mathrm{c}}$ (except around some well-defined directions). That is why we have tilted the sample by an angle $\alpha=30^{\circ}$ with respect to the beam direction.

This geometry makes the system insensitive to a tilt along a direction parallel to the incidence plane for an extraordinary incident polarization. However the tilt is expected to occur at random around the initial molecular axis direction, and then the effect of the linear analyzer is only to reduce the sensitivity of the detection.

3.1.3 Mechanical conditions for the sample holder [12]. - The stresses are imposed by piezoelectric ceramics on which a voltage $V$ is applied. In general, it is difficult to know the exact deformation of the ceramics. One should take into account the elastic reaction of the smectic crystal on the holder and the ceramics. This reaction is proportional to $B$, to the sample area and to the inverse of the sample thickness ; it is to be compared with the rigidity of the ceramics and of the holder. The holder is composed of parts made of brass, expoxy resin and glass. In our set-up we estimate that the largest elastic response of the holder is mainly due to three screws that fix together the two brass parts (Fig. 5a, b). Taking into account the ratio of the elastic moduli and the ratio of the respective surfaces of the screws and of the crystal we find that the rigidity of the holder is larger than that of the crystal only for sample thickness larger than $100 \mu \mathrm{m}$. Typically for a $100 \mu \mathrm{m}$ thickness the reaction of the crystal is of the order of $10 \%$ of the action for a $1 \mathrm{~cm}^{2}$ sample area.

Let us now consider the two types of experiments :

a) Dilation experiment : the deformation threshold for the undulation instability is a displacement threshold independent of the sample thickness. The acting force necessary to produce this instability tends to zero in the limit of large values of $d$ as well as the stresses in the sample. Generally the sample thickness is larger than $100 \mu \mathrm{m}$. In that case, the elastic reaction on the holder being negligible, the applied displacement proportional to the applied voltage is equal to the free-space displacement of the ceramics.

b) Compression experiment : one has to produce a stress larger than the stress threshold (see $\S 2.2 .2$ ) in 
order to observe the tilt instability. It is easier to produce if for thin samples (typically with our ceramics the threshold is obtained with reasonable stresses for thicknesses of some tens of $\mu \mathrm{m}$ at $0.1^{\circ} \mathrm{C}$ off the transition temperature). In that case the acting force must be large and the displacement (as well as the strain in the sample) tends to zero in the limit of very small thicknesses. In that limit it is the force $\mathcal{F}$ applied by the ceramics which is directly proportional to the voltage $V$. Refering to $\S 2.2 .2$ where it is established that the stress threshold $\mathcal{F}_{\mathrm{c}} / S=B_{\perp}$, one could, for very thin samples, measure $B_{\perp}$ directly by measuring the voltage threshold $V_{\text {th }}$.

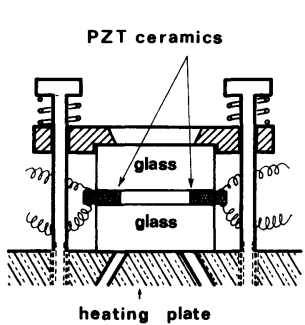

a)

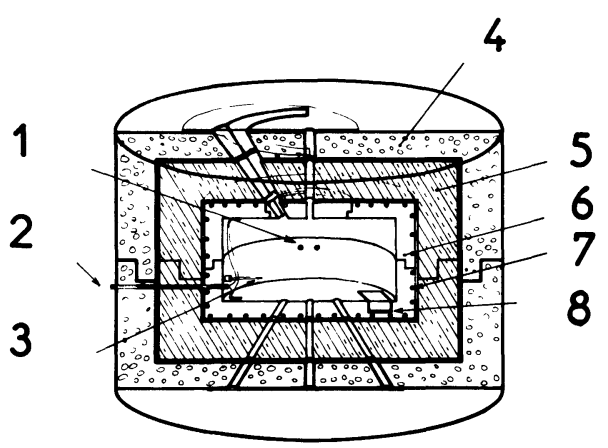

c)

FIG. 5. - $-a, b)$ Schematic of the experimental sample holder a) For the dilation experiment. $b$ ) For the compression experiment. c) The thermal enclosure : 1) electrical supply for the PZT ceramics; 2) differential thermocouple; 3) sample internal temperature probe ; 4) polyurethane foam ; 5) glass fibers in epoxy resin 6) copper ; 7) coaxial heating wire ; 8) platinum sensor for thermal control.

The transition between these two limiting regimes is defined by the comparative rigidities of the holder and of the sample. In practice as has been shown previously, the two types of experiments are performed using the same kind of holders and then the thresholds in both cases will be measured directly by the voltage thresholds.

3.2 THE EXPERIMENTAL SET-UP. - The set-up is essentially the same for both experiments [12]. The samples are prepared between glass plates $4 \mathrm{~mm}$ thick. flat to better than $\lambda / 8$ and coated with a suitable aligning agent to obtain the homeotropic geometry : HTAB (Hexadecyl trimethylamoniumbromide) in chloroform $\left(10^{-3}\right.$ mole per liter) (this compound is also called CTAB).
For sample thickness larger than $250 \mu \mathrm{m}$ (used in the dilation experiment) the spacers are two rectangular piezo-electric ceramics. These ceramics are chosen to have the same thickness within 1 or $2 \mu \mathrm{m}$. A metallic holder is used to maintain, with weak springs, the spacers between the glass plates (Fig. $5 a$ ).

For samples thinner than $200 \mu \mathrm{m}$ (used in both experiments) the glass plates are mounted in a FabryPérot-like holder with no spacers (Fig. $5 b$ ). The piezo-electric ceramic has an annular shape and is placed between the upper plate and the metallic holder. Each part is held with an epoxy resin. This set-up enables us to adjust the parallelism of the glass plates to better than $10^{-4} \mathrm{rad}$. This is especially important to avoid, as far as possible, any hydrodynamic flow in the central part of the sample.

The ceramics have a typical linear extension coefficient of $4.21 \AA /$ volt at $20^{\circ} \mathrm{C}$, with a relative temperature variation of $2 \times 10^{-3} /{ }^{\circ} \mathrm{C}$. A precise value of this coefficient is given for each ceramic by the maker and is not controlled by us. These ceramics are driven by a Hewlett-Packard HP-3320 function generator followed by an ATNE $\left({ }^{2}\right)$ amplifier. We apply sequences of square pulses to the ceramics, the period of which is adjusted with respect to the response time of the detected optical signal. The rise-time of a step voltage measured on the ceramics is less than $10 \mu$ s from 0 to 500 volts. The light source is a model 124 SpectraPhysics $\mathrm{He}-\mathrm{Ne}$ laser, with beam power attenuated and kept under $3 \mathrm{~mW}$ on a $0.5 \mathrm{~mm}^{2}$ area in order to reduce thermal gradients produced in the sample (in most cases the beam power is less than $0.5 \mathrm{~mW})$. The incident beam is slightly convergent (with an angle of $10^{-2} \mathrm{rad}$ ) in order to reduce the illuminated area. The uncertainty produced in the determination of $q$ is much less than other experimental errors. The scattered light is detected by a BPX 25 phototransistor with a frequency cut-off of $30 \mathrm{kHz}$. The photocurrent is observed on a D-C coupled CRT.

The sample is heated in a thermal enclosure (Fig. 5c) in which the lower part and the upper part have separate heating adjustments. The vertical gradient, measured between the top and the bottom with a Copper-Constantan differential thermocouple, can then be minimized. The sample temperature is measured by a Copper-Constantan thermocouple of $70 \mu \mathrm{m}$ diameter located directly in the liquid crystal $1 \mathrm{~mm}$ away from the laser beam in samples thicker than $200 \mu \mathrm{m}$. For the thinner samples the same thermocouple is placed in the holder $0.5 \mathrm{~mm}$ away from the lower glass plate. Another differential Copper-Constantan thermocouple is used in thick samples to measure the radial gradient inside the liquid crystal. The temperatures are measured with respect to an ice-point reference, Kaye 150 , stable to $2 \times 10^{-3}{ }^{\circ} \mathrm{C}$ per hour. with a HP 3420 differential

( $\left.{ }^{2}\right)$ ATNE : Application des techniques Nouvelles en Electronique, Z.I. de Courtabœuf, 91400 Orsay. 
microvoltmeter. For thick samples, measurements indicate that the vertical temperature difference between the top and the bottom of the oven can be adjusted to less than $2 \times 10^{-2} \mathrm{o} C$ and the radial gradient is of about $2 \times 10^{-2} \mathrm{o} / \mathrm{mm}$. For thin samples the vertical gradient is estimated to be reduced in proportion to the thickness, and the radial one identical to the one of the thick samples. The oven is mounted on a holder which can rotate around two axes : one is normal to the incidence plane and the other one is normal to the sample. That allows, as we have previously seen, a positioning of the wavevector $\mathbf{q}$ extremity on the scattering circle. The polarizers are Nicol prisms; the analyzer used in the dilation experiment is an $\mathrm{HN} 32$ Polaroid.

3.3 ObSERVATIONS AND RESUlts. - Some of the following results have already been presented [14] or published in a shorter form $[1,3,13,14,15]$.

\subsubsection{Undulation instability of layers. -} 3.3.1 a) Direct observation of the deformed structure. - At a fixed temperature far from the nematic transition we observe under a polarizing microscope the area that will be illuminated by the laser beam; we apply square voltage pulses at low frequencies ( $\sim 0.5 \mathrm{~Hz}$ ) to the ceramics. Even when no voltage is applied to the ceramics we may already see ripples. generally around large surface defects (see Fig. 6).

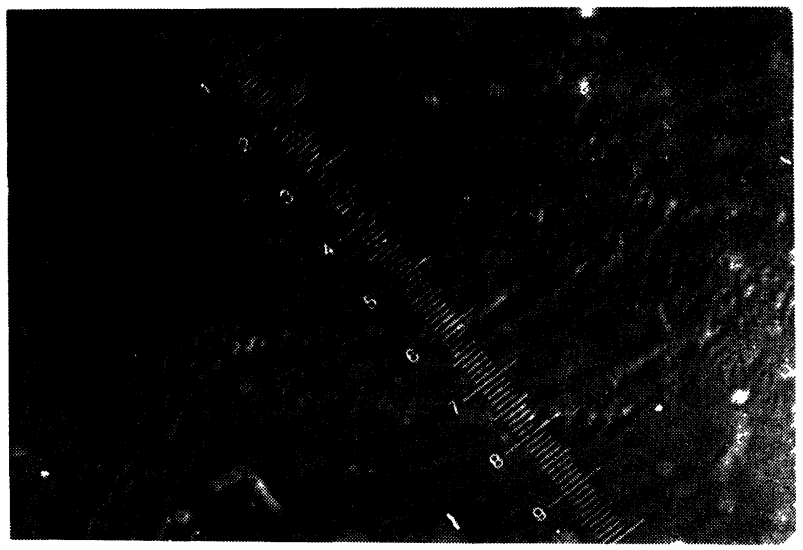

FIG. 6. - Static undulations appearing close to defects or irregularities on the vicinity of the glass plates limiting the sample. These undulations are responsible for the scattering appearing as portions of circles. (ref. [11]).

When the voltage is applied nothing is observed until a certain threshold (typically $30 \mathrm{~V}$ ) is reached for the dilative phase of the signal. Then there appears an array of equally spaced thin lines stationary in space and analogous to a grating with a low contrast (Fig.7a). This pattern is temporary and disappears as the voltage is kept constant. Generally it is wiped off from one side to the other, while some undulations may remain around a defect.

On the other hand. we observe that generally no distortion appears when the voltage falls down to zero.

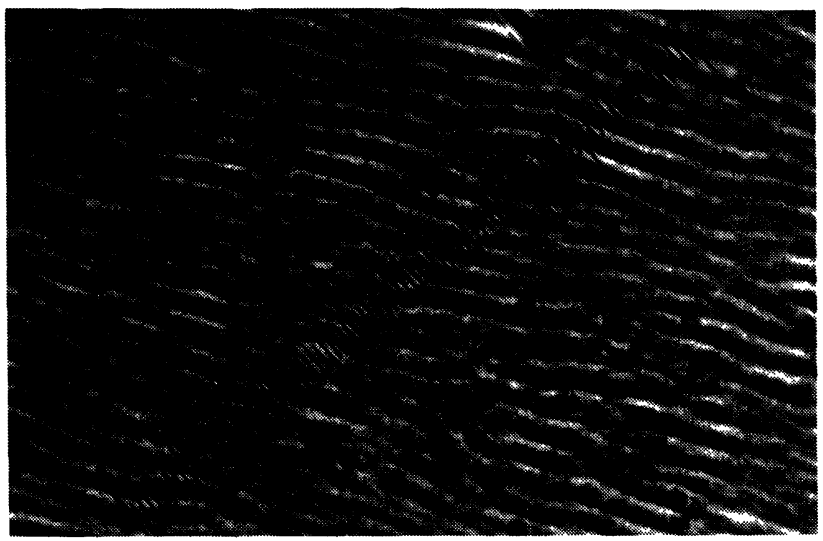

a)

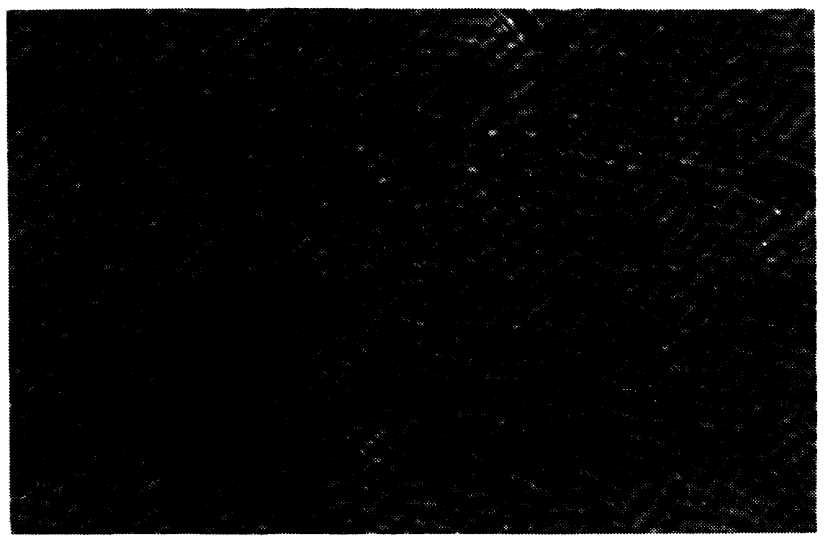

b)

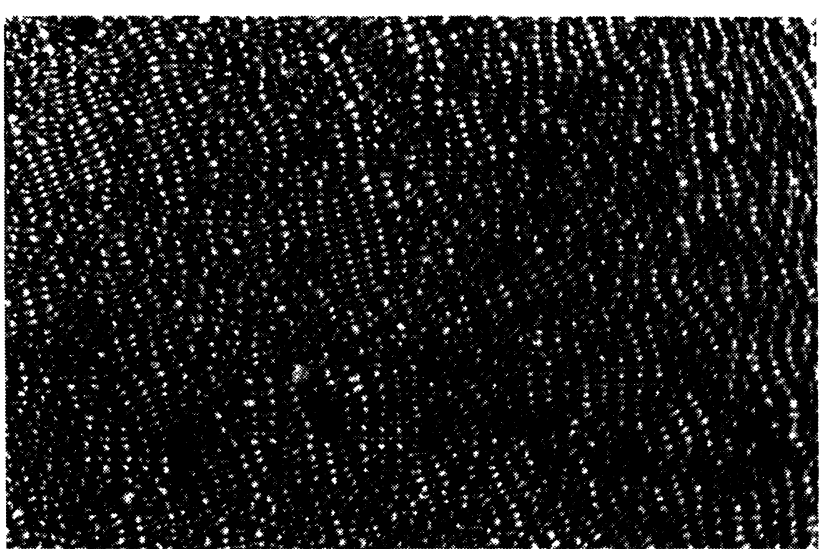

c)

FIG. 7. - Deformations of the smectic structure submitted to a dilative stress and observed in the direction of the optical axis (normal to the layers) under a polarizing microscope. $a$ ) Single grating : undulations of wave-vector $q_{\mathrm{c}}$ (here the dilation is very high in order to obtain a satisfactory optical contrast, resulting then in a highly distorted grating). b) Double crossed grating observed for a higher stress. Here the angle between the two gratings is different from $\pi / 2$ due to the high value of the applied stress. c) Broken structure composed of focal conic domains. It is obtained for a stress at least ten times higher than the stress threshold corresponding to the undulation instability (single grating). The average size of the domains is 5-7 $\mu \mathrm{m}$ here. 
However if there were some static distortions present due to defects, we observe that when compression is applied (by reversing the voltage), these distortions are suppressed. Moreover if a distortion has been produced by dilation and if we apply a compression before the natural relaxation time we supress this distortion. Increasing the dilative stress, we observe that for a voltage 4 or 5 times higher than the threshold value there appears a double-crossed array of thin lines (see Fig. 7b) which disappears after a longer time, evolving back from the double array to a single one which is then wiped off. These two different gratings make a relative angle which is close to $90^{\circ}$ near this second threshold and decreases towards $60^{\circ}$ as the voltage is increased. We again increase the voltage, and we can see (for some samples) an increase, by a factor of 2 to 5 , in the line-spacing, followed, when $V$ is of 10 times the threshold, by a breaking of the structure into small identical regular domains which appear to be composed of focal conics (apparent diameter close to $4-10 \mu \mathrm{m}$ ), see Fig. $7 c$. This broken structure may remain for times ranging from tens of seconds to some hours and could correspond to a storage mode. The duration of this broken structure seems to depend on the applied stress, and to show the existence of a new threshold which is difficult to define and measure. For stresses below this threshold the duration of this structure lasts from some tens of seconds to some minutes. However for high stresses above this threshold the new structure can last from many hours up to some days. That could be due to a destruction of the molecular anchoring at the glass surfaces and might correspond to the configurations described by Parodi [16].

The restabilizing effect of the compression phase is more easily observed after producing a broken structure. Applying a large compressive stress then makes the focal domains disappear. We can then go back to an unperturbed structure by slowly relaxing the applied compression. This effect which is restabilizing could be used as an erasing process for the storage mode.

3.3.1 b) Visual observation of the instability in the light scattering pattern. - We now illuminate the sample with the laser beam. In absence of applied voltage we observe on the screen (see Fig. $8 b, c$ ) the scattering on two portions of circles due to the static undulations still present in every sample due to irregularities on the surface of the glass plates [11].

The width of these circles (the crescent) is directly related to the component of the wave-vector $q$ outside the plane of the layers. The $q_{z}$ component is related to the boundary condition imposed by the plates. For a sample of thickness $d$, the lowest-order spatial mode has a component of $\mathbf{q}$ given by $q_{z}=\pi / d$. Typically for a thickness $d=100 \mu \mathrm{m}$ the angular aperture measured from the center of the sample and corresponding to the spatial width of the crescent would be of order of $10^{-3} \mathrm{rad}$. Experimentally we find

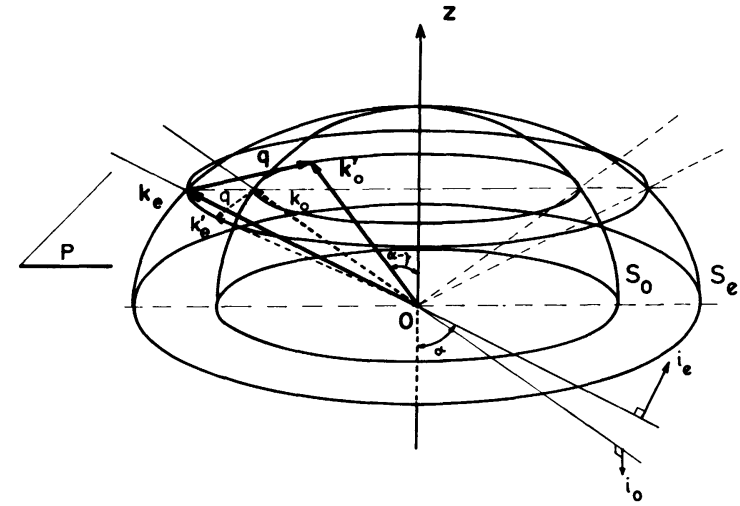

a)

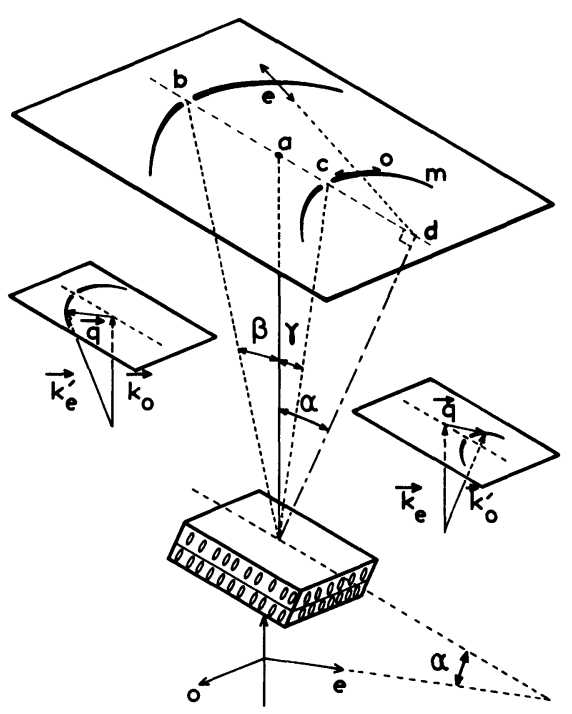

b)

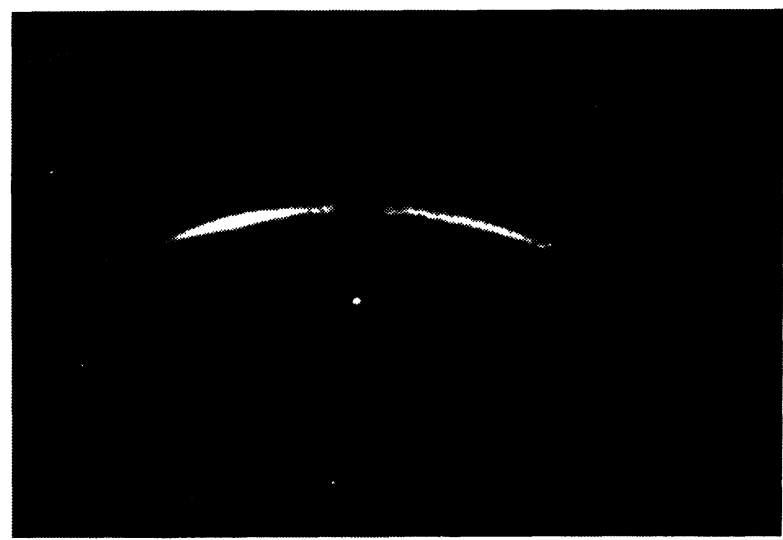

c)

FIG. 8. - Scattering by undulations of layers in a smectic-A positive uniaxial crystal. $a$ ) Distribution of the scattering on concentric cones. For example, the incident ordinarily polarized beam $\left(i_{0}\right)$ - of wave-vector $k_{0}$ is scattered by $q$ lying in a plane (P) normal to the optical axis (along $z$ ). The locus of the scattered wave-vector $k_{\mathrm{e}}^{\prime}$ (with extraordinary polarization) for any value of $q$ is a circle intersection of $S_{\mathrm{e}}$ by $(\mathrm{P}) . b$ ) Geometry of the scattering on a screen parallel to the sample. The scattering is in fact reduced to portions of the circles. That limitation of scattering shows that there exists a cut-off in the wave-vectors $q$ that deform the structure (only the small values of $q$ significantly deform the structure). The position of the extremity of the crescents could give a way to measure the critical wave-vector $q_{\mathrm{c}}$ [12]. $c$ ) Pattern of the scattering on a screen parallel to the smectic planes. The central dot is the laser beam trace. The incident light polarization is ordinary. 
values ranging from $10^{-2}$ rad. for the good quality samples [17] to values around 2 or $310^{-2} \mathrm{rad}$, for the average quality samples. This large difference might be due to spurious scattering by the large defects present in the sample which would excite higher-order $z$ modes. No detectable thickness dependence of the width can be detected for $d$ varying between 100 and $800 \mu \mathrm{m}$ (although for $d<100 \mu \mathrm{m}$ the width of the crescent spreads out). In any case a direct measurement of the width has not been found in agreement with the expected value. If we apply the voltage we observe, above a threshold, a strong scattering restricted to two small parts of the scattering circle (see Fig. 9a, $b$ ). We check that the observed scattered light follows the right polarization selection rules for scattering by a wave-vector lying in the plane of the layers ; it has the characteristics of a selective diffraction by a grating (Bragg diffraction). The increase of light is sudden and lasts for a short time ; the comparison of the voltages shows that it corresponds to the appearance of the simple grating observed under

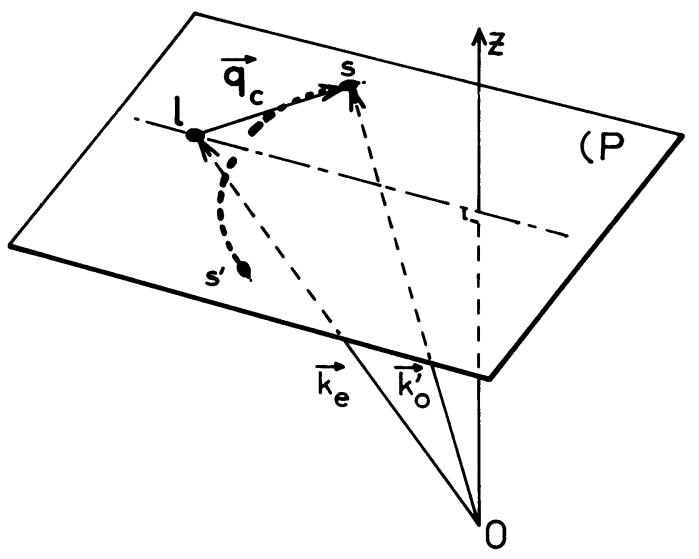

a)

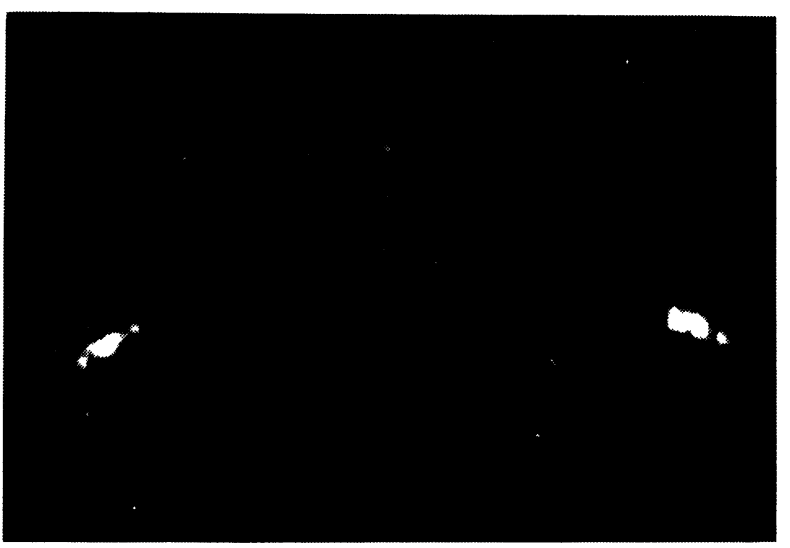

b)

Fig. 9. - $-a$ ) Geometry for the scattering by the undulations of wave-vector $\mathbf{q}_{\mathrm{c}}$ obtained under a dilative stress (single grating). The scattering occurs in two well-defined directions. (The geometry is drawn here inside the crystal.) $\mathbf{k}_{\mathrm{e}}$ is the incident wave-vector, $\mathbf{k}_{0}^{\prime}$ the scattered wave-vector; the subscript $\mathbf{o}$ stands for ordinary and e for extraordinary polarizations. $b$ ) Pattern of the scattering by the undulations, on a screen parallel to the sample layers. The distance between the laser trace and each bright spot is related to the value of $q_{\mathrm{c}}$ microscope. The thresholds for both effects are equal. We increase the voltage and see that the transient intensity of the spots increases sharply. For a voltage 4 or 5 times larger than the threshold the scattering circle splits up into two pseudo-circles intersecting on the incidence plane. This effect lasts a longer time than the former one and disappears by reducing to a single circle. It corresponds to the double-crossed structure. For higher voltages we see a splitting into three circles. The corresponding observation under the microscope has not shown any different structure. These effects could be interpreted as follows : for high dilative stresses the planes of layers are tilted by a finite angle and symmetrically with respect to the initial position. That might correspond to a breaking of the undulation into a zig-zag structure. Thus the two families of planes each give a different scattering pattern. If so, the separation angle between the two pseudo-circles on the observation plane (these pseudo-circles would in fact be two ellipses secant on the incidence plane) could give the limit angle corresponding to the rupture limit of the layers. We find typically an angle of about 0.1 rad. for CBOOA at $74^{\circ} \mathrm{C}$.

For higher stresses one can observe another scattering circle concentric with the previous one and passing by the laser beam trace. This scattering pattern corresponds to the $k_{0} \rightarrow k_{0}^{\prime}$ configuration which leads, in a linear regime, to a zero cross-section, since only the splay mode of deformation is allowed. However in a highly perturbed structure where the undulation occurs not only in one direction but in two orthogonal directions [13], one can conceive that it is necessary to introduce a twist mode by reason of continuity between these two main directions.

For voltages exceeding about 10 times the undulation threshold a strong scattering occurs, as the first voltage step is applied, on a very diffuse cone (half angle of about $0.1 \mathrm{rad}$.), and may last from some tens of seconds to some hours depending on the sample and the voltage. That corresponds to the " broken structure " observed under microscope, and the measured angle is consistent with scattering by objects of about 5-7 $\mu \mathrm{m}$ which are the observed focal domains.

Effect of a compressive stress. - Reversing the voltage in order to apply a compression we note that in the presence of scattering by static undulations only, both the scattered intensity and the width of the pattern decrease strongly. Similary, applying a compressive stress just after a dilative one which produces an undulation, results in a sudden decrease of the scattered intensity.

3.3.1 c) Time analysis of the transient increase in the scattered light. - At a fixed temperature, we determine approximately the position of the Bragg diffraction spots (at $q_{\mathrm{c}}$ ) on the scattering circle by gently touching the sample; we increase its intensity by simultaneously rotating the sample holder, if necessary. Then the phototransistor position is adjusted along this direction, behind a properly oriented 
analyzer. Generally it is convenient to use an extraordinary incoming polarization so that the analyzer direction is tangent to the inner circle. When no voltage is applied on the ceramics the light scattered by static undulation of layers gives rise to a photosignal background. Moreover, we have observed in good quality samples which show a less intense scattering by static defects, a small amount of fluctuating signal due to the thermally excited undulations that have recently been observed in another experiment [17]. We apply the square voltage pulses (typical value : 40 volts; half period : $2 \mathrm{~s}$ ) producing a dilative stress. A transient photo-signal is then observed. We check that this signal disappears when the analyzer is rotated in the plane normal to the optical axis by $90^{\circ}$, thus meaning that it follows the polarization selection rules of scattering by layer undulations [10]. The optical signal generally appears as a sharp peak above a plateau (see Fig. 10). The peak has a short rise-time (of about 1-2 ms). and an exponential-like decay-time (typical value $15-20 \mathrm{~ms}$ ), The plateau is in fact a long-time decaying signal (times ranging from some $50-100 \mathrm{~ms}$ to some seconds). In order, now, to understand this complex signal we must carefully analyze its dependence on the applied voltage.

3.3.1 d) Voltage dependence of the photo-signal amplitude. - Increasing the voltage continuously

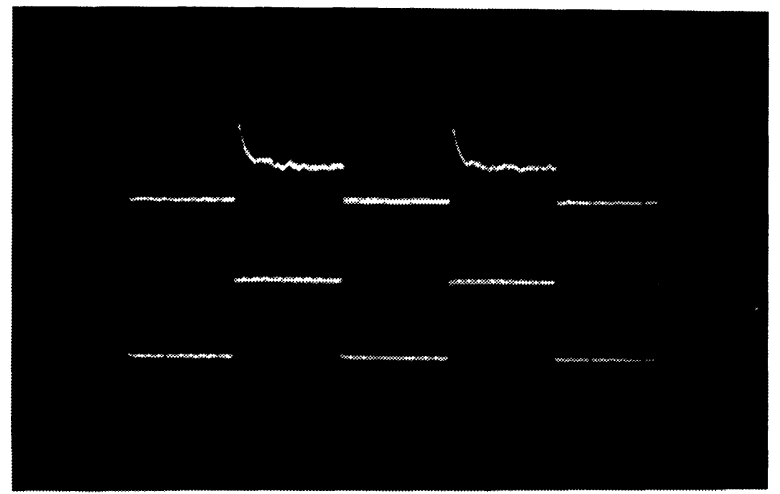

a)

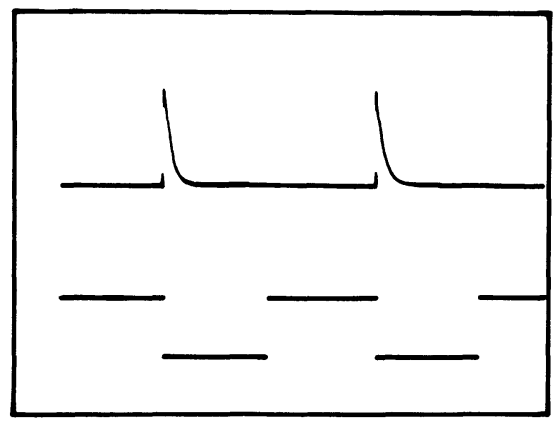

b)

FIG. 10. - Photo signals detected on the direction of the two bright spots due to scattering by the undulation with the wavevector $q_{\mathrm{c}}$. (The lower-trace is the excitation signal.) $a$ ) The signal is composed of a peak superimposed on a plateau. (Existence of two very different exponential decays.) $b$ ) Only the peak is present (single exponential decay). from zero we note that : first, the small plateau increases from zero, then the sharp peak is superimposed on it. Still increasing the voltage, we observe that the peak amplitude increases suddenly and more rapidly than the plateau. For some samples we observe that only the peak is present. In a first approach we choose to measure the peak and the plateau amplitude separately. By peak amplitude we mean here the part of the signal measured above the plateau. For a good sample, a typical plot of the peak and plateau amplitudes versus the voltage is shown figure 11 . We see that the peak curve clearly shows a threshold, followed by a linear part. This threshold is the one corresponding to the observation of the grating. For even higher voltage a saturation effect is observed. The plateau curve shows a smooth increase followed by a sharp variation and by a saturation effect. The sharp variation indicates the existence of a threshold different from that of the peak. However, on most of the average-quality samples the threshold of the peak curve is replaced by a break of slope in a curve which shows a small continuous increase of amplitude from the origin (see Fig. 12). Furthermore, observations on different samples show that the relative magnitude of the plateau varies strongly from sample to sample and indeed, this plateau may not even appear. We have measured on the same sample the voltage dependences for both signals before and after submitting the sample to sequences of dilation-compression for three hours. The results show (see Fig. 11) that for any voltage the plateau has decreased while the peak amplitude remains almost constant.

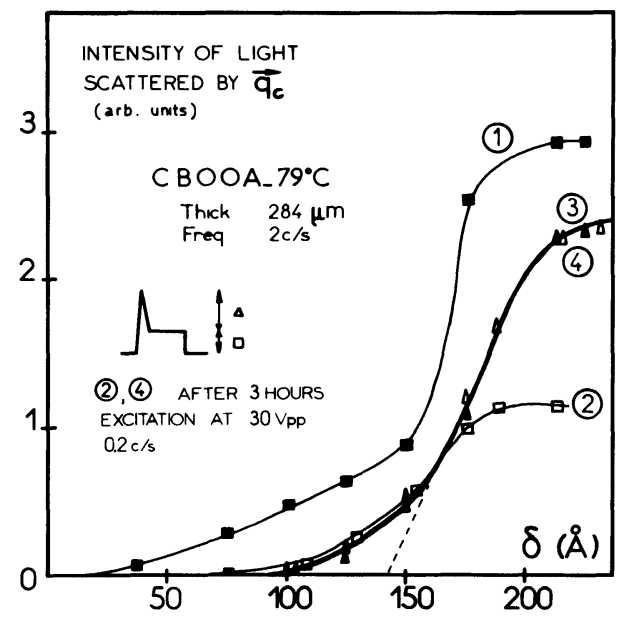

FIG. 11. - Amplitudes of the peak and of the plateau before (respectively 3 and 1) and after (resp. 4 and 2) the application during 3 hours of sequences of compression dilation, with an amplitude higher than the threshold value. The peak curve remains almost unchanged while the plateau curve varies strongly.

We notice too, that the linear part of the peak-curve corresponds to scattering on a single circle, while the onset of the saturation part corresponds to the splitting into two circles (double-crossed gratings).

From all these observations we identify the peak part as due to the undulation instability relaxed by a 
simple mechanism, while the plateau appears to be more complicated to interprete. In a simple approach this platea $u$ would be due to the instability relaxed by a very slow mechanism (its relaxation time corresponds to the transit time of dislocations traversing the area illuminated by the beam). It could reflect a more complicated situation including the previous one and the stimulation of defects by the stresses. Since this plateau does not appear for every sample we choose to analyze only samples showing a quasi-unique relaxation process. On the other hand it is observed that the plateau contribution is relatively weak and that it appears after some non-sharp threshold higher than that of the peak part. It is found experimentally that we can define the threshold of the instability by measuring only that of the peak part. The error on the absolute value for the threshold then lies within other experimental errors less than $5 \%$. The peak curve shows a linear dependence as expected from the instability signal, and we define the threshold $V_{\mathrm{th}}$ as the intersection of this linear part with a straight line tangent at $V=0$ to the part of the curve before the linear increase. On our typical samples, $V_{\mathrm{th}} \simeq 30 \mathrm{~V}$, at $T=75^{\circ} \mathrm{C}$ in CBOOA.

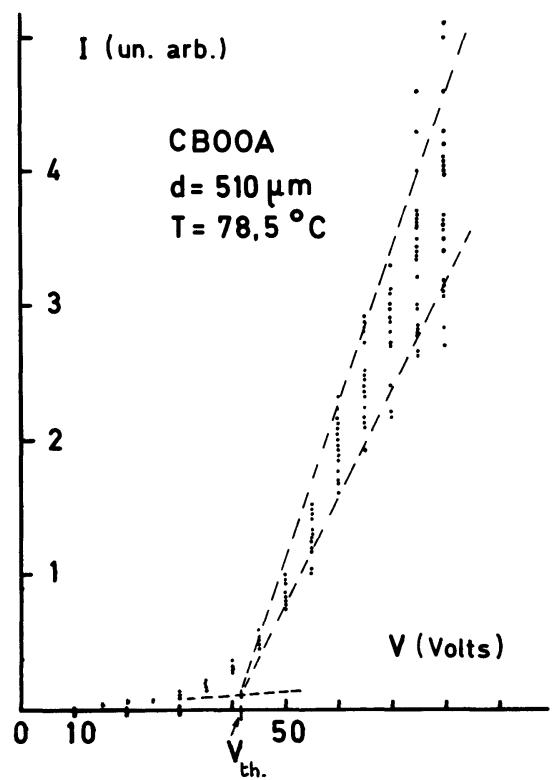

Fig. 12. - A typical dependence of the photo-signal amplitude (peak part only) on the voltage applied on the piezo-electric ceramics which produces the dilation (the dilation $\delta=k . V$ with here $k \simeq 4.7 \AA /$ volt). $V_{\text {th }}$ is the threshold of the instability. The part of the curve before the threshold of the instability may correspond to a stimulation of the static defects by the stress.

3.3.1 e) Voltage dependence of the peak decay time. - As the voltage is varied we observe that the $\overline{\text { peak }}$ decay time varies while the plateau decay time remains apparently constant.

For the part of the amplitude curve of the peak before threshold (when it exists) we see on figure 13 that, as $V$ is increased from zero, the decay time varies typically in a quasi-linear way from $80-100 \mathrm{~ms}$ down to $5-10 \mathrm{~ms}$ close to the threshold. Then it increases smoothly from this last value up to $15-35 \mathrm{~ms}$ close to the onset of the saturation part of the curve. From this point it increases sharply up to some $100 \mathrm{~ms}$. The absolute values for the decay time may vary strongly from sample to sample but the relative behaviour remains similar. For less good samples we have observed that the peak and the plateau decay times may become almost comparable, the last one decreasing down to typically $200-400 \mathrm{~ms}$. A similar behaviour is observed if the phototransistor is not exactly positioned on the central part of the scattering circle width.

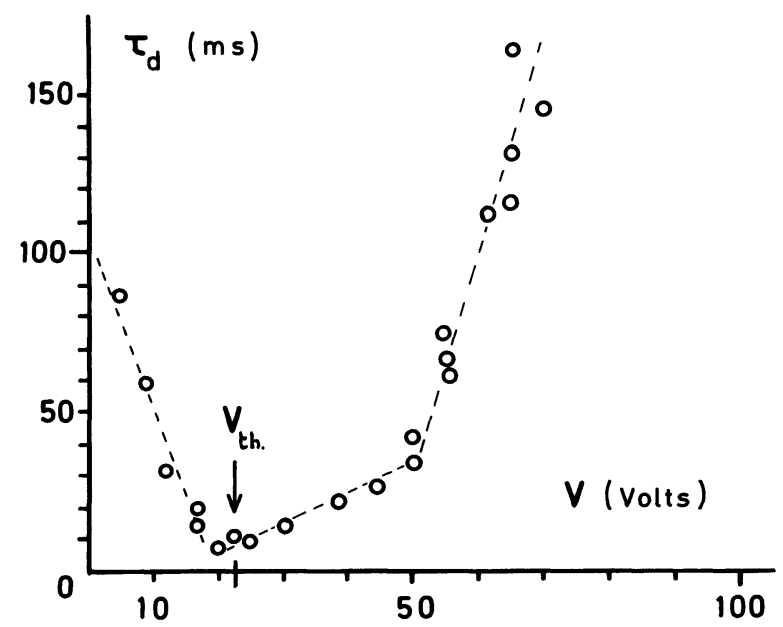

Fig. 13. - Decay time $\tau_{\mathrm{d}}$ of the photo-signal as a function of the applied voltage (i.e. the dilation amplitude) on the ceramics. The minimum corresponds to the onset of the instability. The sudden change of slope (for $V \sim 50$ volts) corresponds to the onset of the double crossed grating.

3.3.1 $\mathrm{f}$ ) Direct observation of a mechanism responsible for the finite duration of the instability [12]. The lifetime of the deformed structure is limited by a phenomenon that can be observed under microscope using a dark background technique (it can also be observed close to small residual defects inside the sample due for example to striations on the glass plates or around small dust particles). Once the undulation is established one can see in the bulk, between crossed polarizers, light thin lines parallel to the undulation lines, and which move normally to them. This motion seems to occur in a plane parallel to the glass plates. The average length of these lines is of $15 \mu \mathrm{m}$ and in our samples they were separated by about $20-25 \mu \mathrm{m}$. The average speed, roughly measured, ranges from 50 to $100 \mu \mathrm{m} / \mathrm{s}$. When this motion is stopped, for example near a large defect (dust...) we can see a larger line leaving the defect and moving back in the opposite direction. This large line wipes out the undulation and takes a quasi-circular shape as it moves. Its speed is of order of $50 \mu \mathrm{m} / \mathrm{s}$. There are many such lines which anneal the deformation and when the wiping process is at end, the lines meet, and form highly contrasted lines similar to walls (Fig. 14). 
Under compression-dilation sequences these walls can emit thin light lines and disappear. We interpret the thin and large lines as being edge dislocations with a small Burgers vector. In effect near threshold, only a few extra layers are needed to relax the applied stress. This number is of order of $2 \pi \lambda / \mathrm{a} \sim 5$ to 6 layers.

From these observations, using the relationship found for the friction force acting on a dislocation, we can deduce a first value for the mobility of such a dislocation. Starting from the measured speed of $50 \mu \mathrm{m} / \mathrm{s}$, which corresponds to an applied displacement of about $1.5 \delta_{\mathrm{th}}$, and using for the diffusivity of orientation $K_{1} / \eta$ the value $2 \times 10^{-6} \mathrm{~cm}^{2} / \mathrm{s}$ found in another experiment [17], we find that with $K_{1} \simeq 5 \times 10^{-7} \mathrm{cgs}$ (as in nematics), $\eta \simeq 0.25 \mathrm{cgs}$ and $1 / \kappa \simeq 25 \AA$ compatible with a molecular length, a value for the mobility $\mu$ of dislocations :

$$
\mu \simeq 1 \mathrm{~cm}^{2} / \text { dyne.s } .
$$

This value compares well with an estimation by N. A. Clark and R. B. Meyer [2].

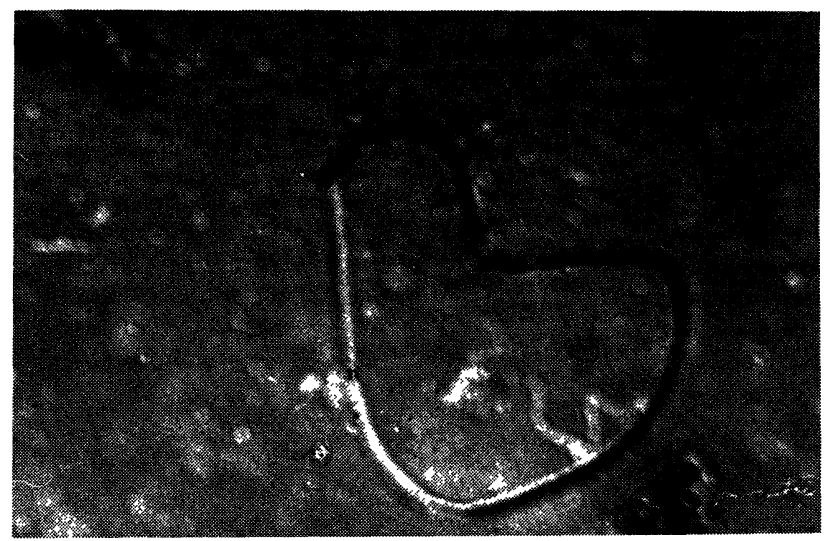

FIG. 14. - Quasi-static wall appearing at the meeting zone of dislocations travelling in opposite directions. These dislocations had just wiped off the structure deformations produced by the dilative stress. Under stresses this wall is able to emit the thin light lines which are suggested to be small burgers vector dislocations (see text).

3.3.1 g) Reproducibility of the threshold determination. Possible influence of structural defects. We have measured, at the same temperature, the instability threshold for various samples and different thicknesses. The results show (Ref. to Fig. 18a) for different thicknesses from $250 \mu \mathrm{m}$ to $800 \mu \mathrm{m}$ a rather large dispersion of the threshold values, whereas no thickness dependence is expected. Our numerous experiments have not shown any systematic variation of the measured thresholds with the sample thickness. On the contrary different samples having the same thickness have given very different values for the threshold. For example we have found that for a given thickness, at the same temperature, the threshold would take the values $10,19.5,30.5,51$ Volts. These numbers seems to be related to the lower one by a ratio close to an integer. This observation has not yet received any satisfactory explanation. We have also noticed that the slope of the linear part of $I(\delta)$ curve varies from sample to sample. In fact, when measuring the peak amplitude versus the applied voltage we generally find, as previously stated, a smoothly increasing curve, then a linear behaviour. For each voltage corresponding to the linear part there exists a large dispersion of the peak amplitude, and the relative dispersion appears almost constant. That leads to an uncertainty in the threshold determination. We are tempted to invoke the influence of the defects present in the sample to explain the poor reproducibility of the results. In effect, as was noticed before, the decay time of the peak varies as the voltage $V$ varies. This decay time is due to some mechanism which tends to relax the applied stress. The simplest and most probable mechanism is the nucleation and motion of edge dislocations. These dislocations can be present in the whole sample or may be nucleated from the edges of the crystal, which are far from the analyzed area. As has been shown, the role of edge dislocations is to relax the stress by adding extra layers. The decay time of the peak could correspond to the motion $($ climb) [18] of the dislocations already present in the sample and which are trapped in the regions of high strain (see Fig. 4c). At threshold, these regions are separated by $2 \pi / q_{\mathrm{c}}$ and the corresponding transit time of dislocations is of order of $50 \mathrm{~ms}$ (see theory). The plateau would correspond to a situation where the stress which has not been totally relaxed by the previous type of dislocation is now relaxed by dislocations traversing the observed area or coming from the edges of the sample (leading to a transit time of order of tens of seconds). The multidomain structure would, furthermore, be responsible for the large dispersion of recorded values of the peak intensity in the linear part. Thus a high-slope variation would correspond to an almost monodomain structure and then give a more reliable threshold value. Static defects which give rise to undulations of layers may also be stimulated by the stress and then contribute to the peak amplitude without having any threshold. That would explain the smoothing of the curve obtained, and even a small decrease of the real threshold.

In conclusion the stimulation of static undulations would be responsible for smoothing of the threshold curve. The presence of large defects such as a polydomain structure would explain the dispersion of the peak amplitude above threshold, and the dislocation motion would correspond to the decay of the instability. With the instability appearing for minimum energy in a monodomain, we might conclude that among many results obtained under the same conditions, the most probable value of $V_{\text {th }}$ is the smallest one. From the threshold value $V_{\text {th }}$ one obtains the penetration length $\lambda$ directly from $\lambda=k \cdot V_{\mathrm{th}} / 2 \pi$ where $k$ is the extension coefficient of the ceramics. The value of $\lambda$ obtained by this way has to be compared with the value which can be deduced from the position of the Bragg spots (see next paragraph). 
3.3.1 h) Measurement of $\lambda$ by the position of the Bragg spots. - The wave-vector $q_{\mathrm{c}}$ of the undulation instability is related to $\lambda$ by $q_{\mathrm{c}}^{2} \lambda d=\pi$ (see section 2.3). Thus by measuring the position of the Bragg spots due to the diffraction by the undulation, relative to the laser trace one can deduce a value for $\lambda[1]$. The experiment has been done using CBOOA (p-cyano-benzilidenep-n-octyl-oxy aniline). The wave-vector is measured according to the method previously described (3.1.1). The dependence of $q_{\mathrm{c}}^{2}$ on the inverse of the thickness $d$ indeed clearly shows a linear variation (Fig. 15). From the slope of this line one can deduce a value for $\lambda$. Typically $\lambda \simeq(14 \pm 2) \AA$ at $73^{\circ} \mathrm{C}$ and $(22 \pm 3) \AA$ at $78{ }^{\circ} \mathrm{C}$. Moreover taking for $K_{1}$ a value close to that of a nematic $\left(K_{1} \simeq 10^{-6}\right.$ dyne $)$ one obtains from the relationship $\lambda=\left(K_{1} / B\right)^{1 / 2}$ an estimate for $B \simeq 2 \times 10^{7}$ dyne $/ \mathrm{cm}^{2}$. This method is a very rapid way to measure $\lambda$ for a given crystal. The values obtained for $\lambda$ by this method have to be compared with those obtained by the direct measurement of the threshold.

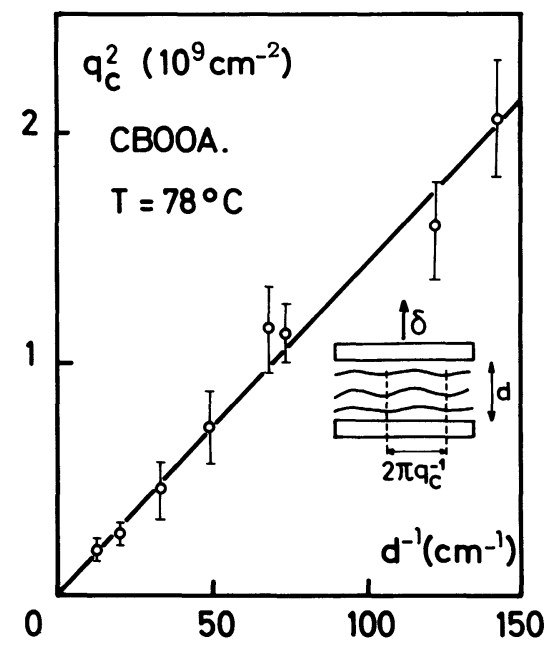

FIG. 15. - Dependence of $q_{\mathrm{c}}^{2}$ deduced from the position of the Bragg spots relative to the laser trace, versus the inverse of the sample thickness $d^{-1}$. The straight line corresponds to $q_{\mathrm{c}}^{2}=\pi / \lambda_{\mathrm{d}}$, with $\lambda=(22 \pm 3) \AA$.

3.3.1 i) Frequency dependence of the threshold. - We measure the threshold for various periods of the applied voltage pulses. The results shown on figure $16 a$ indicate that for low frequencies $(3 \mathrm{~Hz}$ up to $20 \mathrm{~Hz}$ ) the instability and then the applied stress have time enough to relax within half a period. The threshold is then measured by the peak-to-peak amplitude of the pulse. For high frequencies (from $40 \mathrm{~Hz}$ and up) the stresses relax to place their mean zero value at half the voltage value. Therefore in order to reach the threshold one has to increase the applied voltage by a factor of two. The threshold value seems then to relax around a characteristic relaxation frequency of the instability (in the case of the figure : $26 \mathrm{~Hz}$ ).

Dependence for very low frequencies. - When the optical signal is reduced to a peak, experience shows

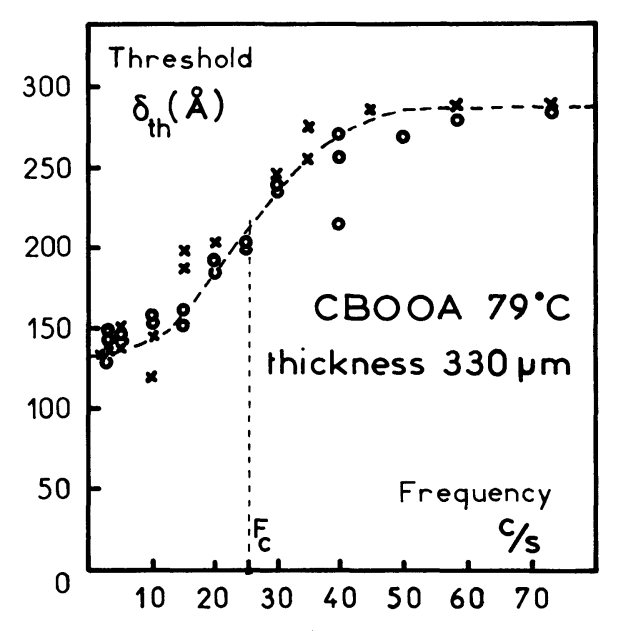

a)

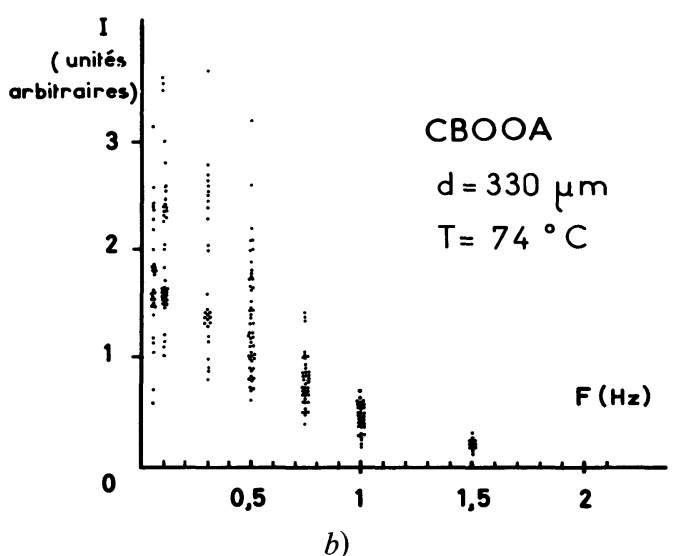

FIG. 16. - $a$ ) Threshold displacement $\delta_{\text {th }}$ as a function of the frequency of the square voltage applied on the ceramics, showing a characteristic relaxation frequency $F_{\mathrm{c}}$ corresponding to the decay time of the instability. b) Low-frequency dependence of the intensity of the scattered light for a dilation $10 \%$ higher than the threshold. Here the excitation signal period is very large compared to the decay time of the instability $\left(\tau_{\mathrm{d}} \simeq 50 \mathrm{~ms}\right)$ and this effect indicates the existence of another, very slow, relaxation process.

that the threshold may vary with the period of the voltage pulses, even when this is very large compared to the decay time of the peak signal. We notice that the $I=f(V)$ curves obtained for periods of $5 \mathrm{~s}$ and $1 \mathrm{~s}$ show an increase in threshold from 17 to 23 volts (here $T=74^{\circ} \mathrm{C}$ ) and a large variation of the slope by a factor of 2 . This effect is more easily observed if we plot the peak amplitude versus the period for an excitation voltage fixed at a value higher by $20 \%$ than the highest threshold value. The result (Fig. 16b) shows that this amplitude decreases strongly as the period increases. This behaviour, which is not correlated with any long-lived signal, could be due to a very slow relaxation mechanism such as a hydrodynamical flow controlled by permeation. This observation indicates that the structure is at equilibrium after times of the order of some 2 or $3 \mathrm{~s}$ following each dilative phase. For this reason we operate with low frequencies in all our experiments $(0.2 \mathrm{~Hz})$.

3.3.1 j) Existence of an instability for wave vectors different from $q_{\mathrm{c}^{\cdot}}-$ Varying the position of the photo- 
transistor along the scattering circle we find that an instability occurs for values of $q$ around $q_{\mathrm{c}}$ and that the threshold presents a minimum value for a wave-vector that we verify as being close to $q_{\mathrm{c}}$ (see Fig. 17). From a monodomain sample we would not expect, in the linear regime, such a dependence, but a unique value at $q_{\mathrm{c}}$. We might explain this as due to some spurious scattering (multiple scattering), or it could to some extent be due to the stimulation of many different modes by means of some non linear-coupling. We also observe that the slope of the linear part of the threshold curves varies strongly as $q$ is varied, being maximum close to $q_{\mathrm{c}}$. A radial dependence on the scattering circle shows that this slope is also maximum at the central part of the pattern. In general the decrease in slope is correlated with the appearance of a strong plateau in the detected signal.

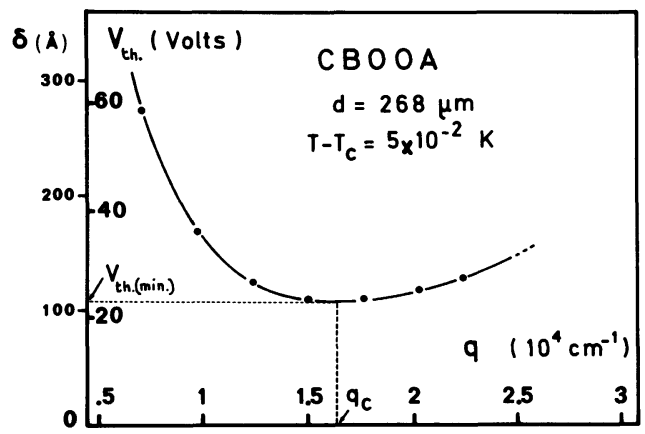

FIG. 17. - Variation of the instability threshold $V_{\mathrm{th}}$ as a function of the wave-vector $q$ (i.e. the position of the photo-cell on the scattering circle). This curve indicates that there might be a large distribution of wave-vectors in the structure deformations. The minimum corresponds to $q_{\mathrm{c}}$.

3.3.1 k) Temperature dependence of the threshold in a rigid smectic-A (CBOOA) [15]. - Measurements of the instability threshold may lead, as has been shown for different samples, to a large dispersion of values for the penetration length $\lambda$. It appears that the defects present in the sample may strongly affect the threshold of the undulation instability. Therefore we take as correct the values of $\lambda$ which are compatible with those simultaneously deduced from the position of the Bragg spots (see $\S 3.3 .1 h$ ). We measure in CBOOA the temperature dependence of the threshold. For every temperature the position of the phototransistor must be readjusted. We plot $V_{\text {th }}$ versus the wave-vector $q$. This method gives both $V_{\text {th min }}$ and $q_{\min } \simeq q_{\mathrm{c}}$. Measurements are made from the smectic-to-nematic transition and down in order to minimize the influence of the drift of $T_{\mathrm{c}}$ on the results. The determination of $T_{\mathrm{c}}$ is made within $10^{-2}{ }^{\circ} \mathrm{C}$ by looking at the appearance of the nematic signal and the disappearance of the scattering circles. The results obtained from experiments made on different samples of the same CBOOA show that (see Fig. 18a) :

- for each set of data the values of $\lambda$ follow the same exponential law. The exponent is found to be close to
$0.165 \pm 0.02$ (Fig. 18a) and $0.15 \pm 0.02$ (Ref. to Fig. 18b) ;

- as previously mentioned, for different samples the measurements of $\lambda$ at the same temperature give values that seem to be related by a ratio close to an integer.

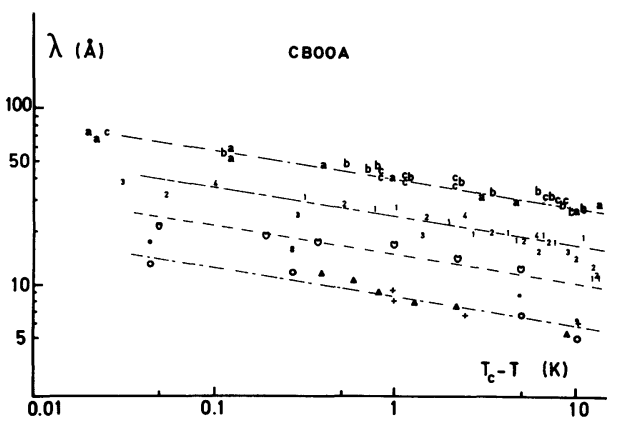

a)

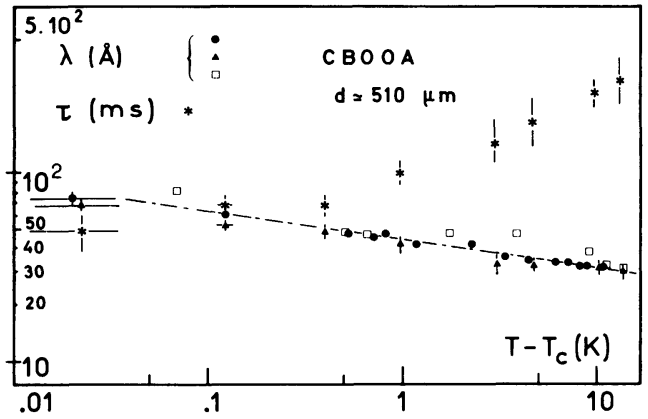

b)

FIG. 18. - Temperature dependence of the de Gennes length $\lambda=(K / B)^{1 / 2}$ close to a quasi-second order smectic-A to nematic transition in CBOOA : a) Set of different curves obtained for different samples (note the quasi-constant spacing between each curve). The slope is the same for each of them and gives an exponent 0.165 . Only the points marked by numbers give values of $\lambda$ compatible whith those deduced from the measurement of $q_{\mathrm{c}} . b$ ) Here the slope gives an exponent close to 0.15 . The decay time $(\tau)$ dependence shows a decrease as the transition is approached.

In conclusion, whatever the absolute value found for $\lambda$, the variation of this value with temperature shows an exponential law with an exponent close to 0.16 . This is to be compared with the value that is expected for the exponent $v / 2(v / 2=0.33$ in a non classical (helium-like) model or $v / 2=0.25$ in a classical (Landau-type) model). Identical results concerning the temperature dependence of $\lambda$ (and B) have also been found recently using the same method [19], and in a light scattering experiment by thermal fluctuations [20].

Discussion : From the analogy with the $\lambda$ transition in Helium the penetration length variation should follow a law of the form $\lambda \sim\left(T_{\mathrm{c}}-T\right)^{-0.33}$. In order to understand the discrepancy with the value deduced from our results, we may first suppose that the transition has significant width $\left(T^{*}-T_{\mathrm{c}}\right)$. In our case we would need a width of the order of $0.5^{\circ} \mathrm{C}$ to find an 
exponent of 0.25 (classical). This is too high when compared with the results obtained on the same compound by P. E. Cladis [21], D. Salin et al. [22] who observed a value for $\left(T^{*}-T_{\mathrm{c}}\right) \lesssim 20 \mathrm{mK}$. In fact, the defects which play an important role might have a temperature dependence and therefore modify the elastic behaviour of the crystal. The observation under a microscope shows that the number of wiping lines increases sharply as $T_{\mathrm{c}}$ is approached while their speed seems to remain constant. Nevertheless we cannot give, up to now, a satisfactory model explaining the role of structural defects.

3.3.1 l) Temperature dependence of the decay time of the instability. - The decay time $\tau_{d}$ shows a significant variation with temperature all over the smectic range. The measurements of $\tau_{\mathrm{d}}$ are made at about $20 \%$ above threshold and the results show (Fig. 18b) a remarkable decrease as the transition to the nematic is approached. This variation does not appear linear on a $\log / \log$ scale, and there exists a temperature zone (at about $0.3-0.4{ }^{\circ} \mathrm{C}$ below $T_{\mathrm{c}}$ ) in which the shape of the optical signals differs from a single exponential. This change in shape might indicate that another relaxation process having a temperature dependence is appearing close to $T_{\mathrm{c}}$. We have not identified this effect.

$3.3 .1 \mathrm{~m}$ ) Undulation instability near a secondorder smectic-A to smectic-C transition : the "Nonrigid smectic $"$. - The compound used here is HOBHA (or 70.7) i.e. : p-n heptyl-oxy-benzilidene-p-n heptyl-aniline, which presents a second-order smectic-A to smectic-C phase transition at $T=72^{\circ} \mathrm{C}$. The samples used are generally $125 \mu \mathrm{m}$ thick. Threshold curves are plotted, and a $q$ dependence on the scattering circle for every fixed temperature is measured the same way as close to the $\mathrm{A} \rightarrow \mathrm{N}$ transition. These curves show a very strong linear increase of the recorded light intensity, and the typical decay time of the signal is of order of $5 \mathrm{~ms}$. The temperature dependence of the minimum threshold shows a decrease as the $\mathrm{C}$ phase is approached (see Fig. 19a, $b$ ) by a factor close to 2 . This result confirms the calculation and shows that a possible tilt of the molecular axis inside each layer tends to decrease the curvature energy of the layers. On the other hand no detectable variation of $q_{\mathrm{c}}$ versus temperature has been measured.

Measurements of the threshold made over the entire smectic-A range show a nearly constant value more than $2^{\circ} \mathrm{C}$ from $T_{\mathrm{c}}$. However, close to the firstorder smectic-A to nematic transition we have found a small decrease. Threshold values in the constant range would give for $\lambda$ a value $\lambda \simeq 76 \AA$ while from the position of the Bragg spots we deduce $\lambda \simeq 19 \AA$. We have not yet explained this large discrepancy and a systematic study of this problem is under way.

The decay time of the signal has not shown any detectable variation over the entire smectic range.

3.3.1 n) Conclusion to the undulation instability
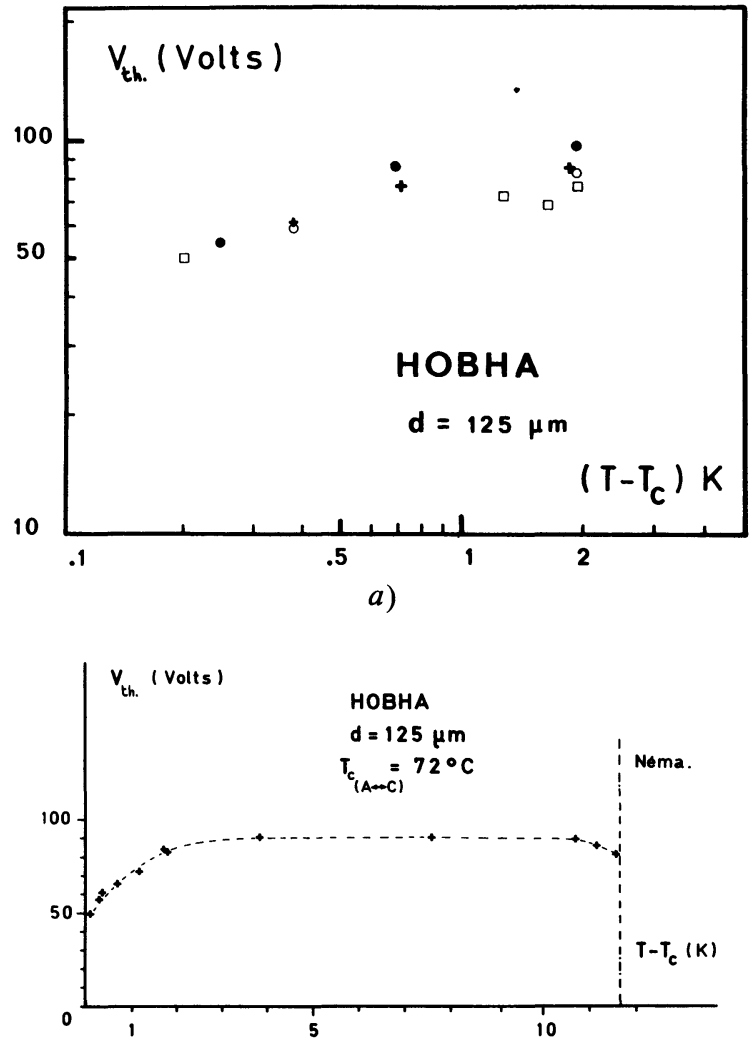

b)

FIG. 19. - $-a, b)$ Temperature dependence of the instability voltage threshold $V_{\text {th }}$ under dilative stress close to a quasi-second-order smectic-A to smectic-C phase transition. Here the value of $\lambda$ is much higher than the one deduced from the spots position.

study. - The undulation instability under dilative stress has been studied in the A phase of the two different types of smectics : rigid and non-rigid smectics (i.e. close to an $\mathrm{A} \rightarrow \mathrm{C}$ transition). The optical signal scattered by the deformed structure is complex. A threshold has been defined using the most significant part of this signal. The study of this threshold as a function of the period of the applied stress, and of the position of the detector has been made together with a study of the dynamics of the photo-signal as a function of the applied stress, and of its period. The results show that the response of the smectic to the dilative stress is strongly dependent, and in a complex way, on the details of the crystal structure, and on the dynamics of the different defects present in the sample. The decay of the instability could be explained by a relaxation of the applied stress by the motions of dislocations. Using simple models we have deduced a first value for the mobility of small dislocations.

On the other hand the dispersion of the threshold values, and the exponent of the $\lambda(T)$ variation have not received any satisfactory explanation.

We have briefly mentioned the possible role of defects. There may be some other reasons to explain the results obtained :

- The role of impurities may not be negligible. These impurities coming from chemical dissociation 
of the molecules can contribute by modifying the macroscopic modulus $B$, or if they are homogeneously spread within the layers they may contribute by altering the Frank elastic constant $K_{1}$.

- Hydrodynamic flow :

This flow is produced under the stresses when the plates are not exactly parallel. It corresponds to a slipping of layers and is not controlled by permeation. It may be responsible for a new type of instability [12] which has been observed under the microscope especially close to the $\mathrm{A} \rightarrow \mathrm{C}$ transition. This instability which appears as an undulation of small wavevector $\mathbf{q}\left(q \sim q_{\mathrm{c}} / 50\right)$ seems to result from a coupling between the molecular orientation and the flow, and is under study at the present time.

\section{- Layer configuration :}

The compound used for the rigid-smectic model is CBOOA which presents a double layered structure. The molecular length is of about $26 \AA$ while the interlayer spacing is of about $35 \AA$. Therefore molecules are supposed to be alternatively slightly displaced with respect to each other along the molecular axis [12]. This configuration might be temperature dependent and induce a non-trivial temperature dependence on the elastic constants.

3.3.2 Tilt instability of molecular axis under compressive stress. The non-rigid smectic [3]. - This instability could, in principle, be observed in any smectic-A phase, provided high enough compressives stresses are applied (pressures of the order $10^{6}$ dyne $/ \mathrm{cm}^{2}$ ). Such stresses are difficult to develop in our experimental set-up and our study is restricted to a region close to a quasi-second-order smectic-A $\rightarrow$ smectic-C transition, and with samples about $20 \mu \mathrm{m}$ thick.

3.3.2 a) Observation of the photo-signal due to the tilt instability. - In order to identify the signal due to the tilt of the molecular axis, two phototransistors are placed, one on the transmitted beam direction, and the other in the direction of the Bragg spots due to the undulation instability (see Fig. 20a). Square voltage pulses of $1 \mathrm{~s}$ duration are applied to the ceramics, giving compression-decompression sequences. The decompression phase corresponds to a dilation because the period is large compared to the time needed for the dislocations to relax the applied stresses. The temperature being fixed at about $0.5^{\circ} \mathrm{C}$ above the transition temperature $T_{\mathrm{c}}$, a signal first appears for a voltage of order 60 volts in the Bragg direction for the dilation phase of the pulses. Increasing the voltage makes a signal appear on the transmitted beam direction, as a sharp peak with an exponentiallike decay, for a voltage $V_{\mathrm{s}} \simeq 200$ volts (both signals, simultaneously recorded, are shown figure $20 b$ ). This last signal (Fig. 20c) is very reproducible from pulse to pulse (within $5 \%$ ) indicating that the molecular tilt is occurring either in a well-defined and constant direc-

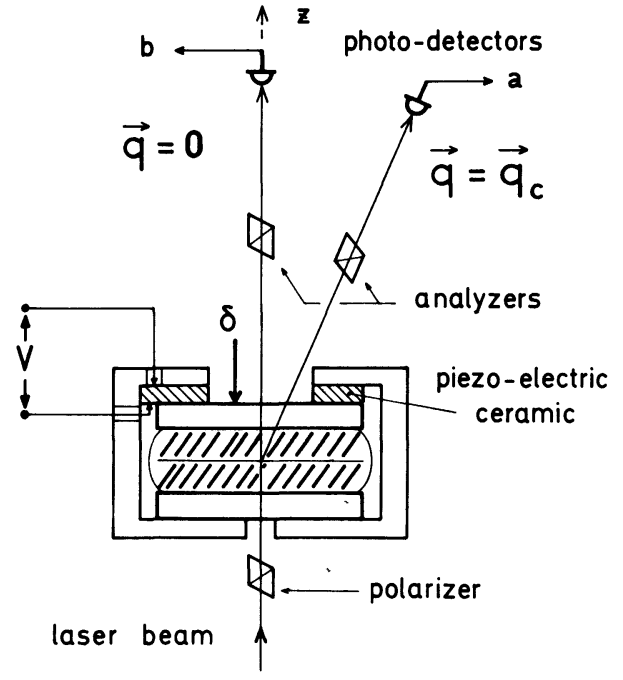

a)

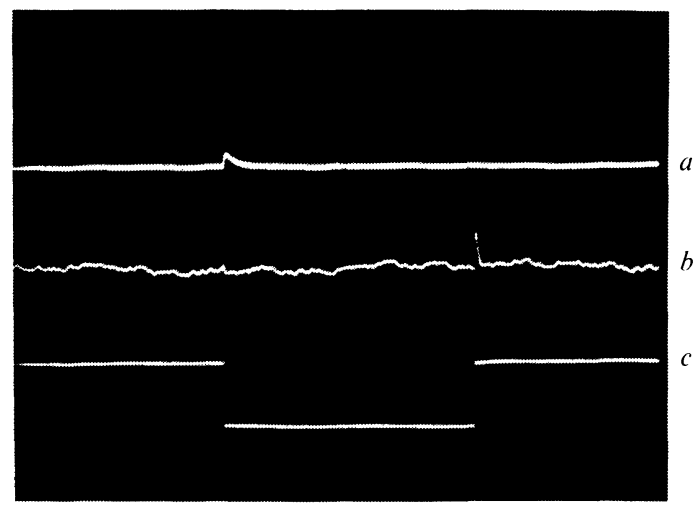

b)

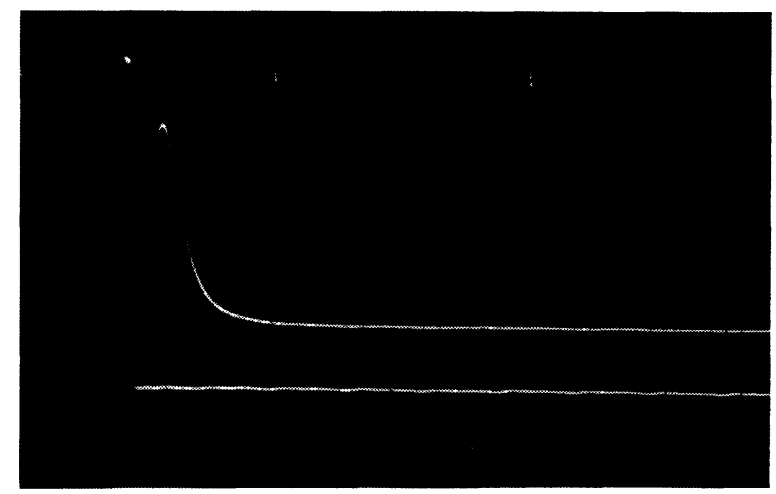

c)

FIG. 20. - Tilt instability of the molecules under a compressive stress. a) Schematic diagram of the set-up fixed for simultaneous observation of the instabilities signal under dilation and compression. $b$ ) For a square voltage applied on the ceramics (trace c), the signal due to the undulations of layers appears for the dilative phase (trace a) while the signal due to the tilt of molecules appears for the compressive phase (trace b). $c$ ) Typical signal due to the molecular tilt (the width at half height is of about $2 \mathrm{~ms}$ ).

tion, or in random directions but within domains smaller than the scattering volume.

3.3.2 b) Voltage dependence of the signal ampli tude. - The signal always appears as a peak showing 
essentially a single decay time. We measure the peak amplitude as a function of the voltage applied to the ceramics. From the threshold $V_{\mathrm{s}}$ where the peak emerges out of fluctuations the variation is found linear until a second value $V^{*}$. Beyond $V^{*}$ the slope increases sharply (see Fig. 21). Observation under the microscope between crossed polarizers shows a large homogeneous increase of light for the compression phase when the voltage is between $V_{\mathrm{s}}$ and $V^{*}$. Above $V^{*}$, for the same phase the crystal structure appears under the microscope as multidomains (mosaics) of well-differentiated illumination. (This structure, which strongly modulates the light with the applied voltage period, could be used in a light modulator device.)

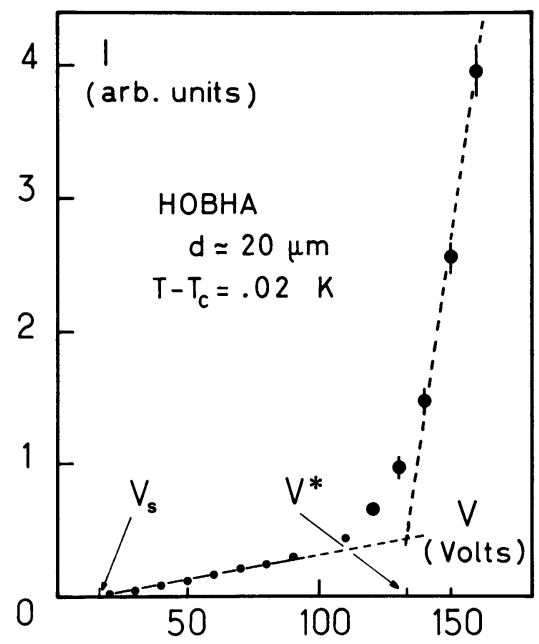

FIG. 21. - Intensity of light transmitted through crossed polarizers as a function of the voltage $V$ applied on the ceramics. $V_{\mathrm{S}}$ is the tilt instability threshold while $V^{*}$ indicates a breaking of the structure into domains.

As has been mentioned previously, for thin samples (less than about $100 \mu \mathrm{m}$ ), the elastic reaction of the crystal on the mechanical holder can be large.

In this limit of zero displacement, the applied voltage is proportional to the threshold stress $B_{\perp}$. We have seen also that, above threshold, the amplitude of the tilt angle $\varphi_{0}$ should be such that $\varphi_{0}^{2} \sim\left|X-X_{\mathrm{c}}\right| / X_{\mathrm{c}}$ with the notation of $\S 2.5 .2$. Since for the adopted scattering geometry the intensity $I$ is of the form $I \sim \varphi_{0}^{2}$, we expect the intensity dependence on the voltage to be :

$$
I \sim \frac{V-V_{\mathrm{s}}}{V_{\mathrm{s}}}
$$

Experimental results show a linear increase between $V_{\mathrm{s}}$ and $V^{*}$. We then conclude that the threshold $V_{\mathrm{s}}$ corresponds to the onset of the molecular tilt instability. The second threshold $V^{*}$ would correspond to the breaking of the layered structure.

In the limit of small sample thickness (and of strong elastic reaction of the smectic) we do not expect in our set-up to observe any thickness dependence of the instability threshold. Indeed, threshold measurements have not shown any thickness dependence, thus confirming our estimate of a strong elastic reaction due to the crystal. Let us now estimate $B_{\perp}$ using the relationship established in $\S 2.3 .2: \mathcal{F}_{\mathrm{c}} / S=B_{\perp}$. The force $\mathcal{F}$ exerted by the ceramic must be calculated from the piezoelectric coefficient expressing the stress at zero deformation of the ceramic. This coefficient is not available and we estimate the force $\mathcal{F}$ by simply using the Young modulus $Y$ of the ceramics $\left(Y \simeq 10^{12}\right.$ dyne $\left./ \mathrm{cm}^{2}\right)$ and the extension coefficient $k$ $(k \simeq 4.2 \AA /$ volt $)$. We assume that the force $\mathcal{F}$. exerted by the ceramic for a zero extension in the presence of the applied voltage is equal to that exerted in extending the ceramic with the same applied voltage in the absence of mechanical reaction. In this estimation we take into account the fact that the area of the ceramic is of 2.5 times smaller than that of the crystal.

We obtain at $\Delta T \simeq 1^{\circ} \mathrm{C}$ from the transition where the voltage threshold is $V_{\mathrm{s}} \simeq 400$ volts (see Fig. 21) :

$$
B_{\perp} \simeq \frac{Y}{2.5} \cdot \frac{k V_{\mathrm{s}}}{e} \simeq 10^{8} \mathrm{dyne} / \mathrm{cm}^{2}
$$

a value which is comparable to that of $B$ and seems one order of magnitude too large. This discrepancy could come from the fact that the ceramics work in fact with a small residual displacement (the reaction of the crystal being over-estimated or the rigidity of the holder being under-estimated).

3.3.2 c) Dynamics of the tilt instability signal. A typical signal obtained $10 \%$ above threshold $V_{\mathrm{s}}$ is shown in figure $20 \mathrm{c}$. Generally no detectable delay is observed before the leading edge. The rise-time is of order of $0.6 \mathrm{~ms}$, and shows no variation with the applied voltage. The decay is generally exponentiallike and the associated decay time $\tau_{\mathbf{d}}$ of order of some ms, varies by a factor of two between $V_{\mathrm{s}}$ and $V^{*}$, then increases sharply from some ms up to some hundreds of ms above $V^{*}$.

The mechanisms responsible for the finite duration of the instability may be very similar to those suggested for the undulation instability :

a) the deformation disappears when the stress is relaxed by elimination of layers. That corresponds to the climb of edge dislocations, towards the edges of the illuminated area, and the associated time is of order of some ms ;

$b$ ) the hydrodynamic flow controlled by permeation. It can correspond to short relaxation times for thin samples;

c) possible impurities segregated between layers inducing defects.

Notice that, contrary to the case of undulation instability no trapping of dislocation equivalent to that in the undulated structure is present here, in principle, since the stresses are uniform throughout the sample. 
3.3.2 d) Temperature dependence of the threshold close to a smectic-A $\leftrightarrow$ smectic-C transition. - We observe, as the $\mathrm{A} \rightarrow \mathrm{C}$ transition is approached, an important variation of $V_{s}$, while the second threshold $V^{*}$ remains almost constant and disappears. Since we expect a critical behaviour for the tilt instability threshold, this indicates that $V_{\mathrm{s}}$ indeed corresponds to this instability. The transition temperature $T_{\mathrm{c}}$ is defined as the temperature for which, in absence of stress, an increase of transmitted light above noise fluctuations indicates the onset of the $\mathrm{C}$ phase. The transition $T_{\mathrm{c}}$ is defined in such a way to better than $2 \times 10^{-2}{ }^{\circ} \mathrm{C}$.

The compounds used here is HOBHA (or 70.7 : p-n heptyl-oxybenzylidene-p-n-hexyl-aniline) which exhibits a transition temperature drift of order $10-20 \mathrm{~m} \mathrm{~K} /$ hour. Therefore measurements are made raising the temperature from $T_{\mathrm{c}}$ in order to diminish the relative error on the temperature definition. The results obtained with sample thickness from $6 \mu \mathrm{m}$ to $125 \mu \mathrm{m}$ are :

- at a given temperature the threshold $V_{\mathrm{s}}$ does not depend on the thickness $d$, confirming that the elastic reaction due to the crystals makes the mechanical system work at nearly zero displacement.

- generally the variation law of $V_{\mathrm{s}}$ (and so of $B_{\perp}$ ) versus $\left(T-T_{\mathrm{c}}\right)$ is quasi-linear, giving an exponent close to one, which then would agree with the value of $\gamma$ in a mean-field theory (Fig. 22);

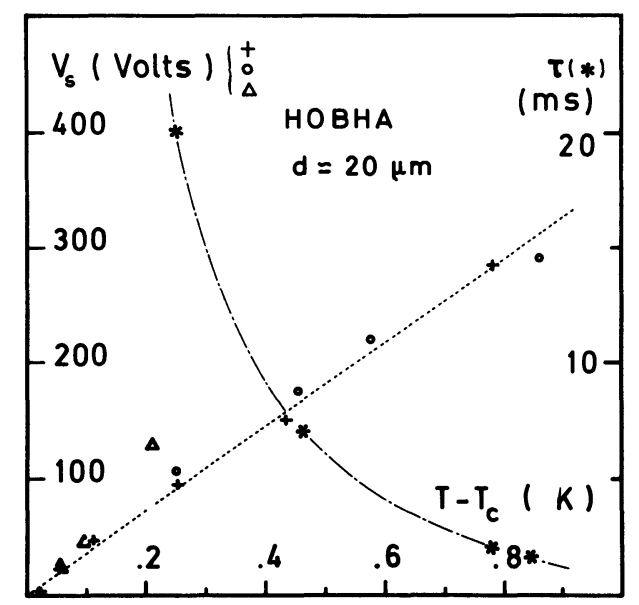

FIG. 22. - Temperature dependence of the voltage threshold $V_{\mathbf{S}}$ close to a quasi-second-order $\mathbf{A} \rightarrow \mathrm{C}$ phase transition. The variation is quasi-linear giving the value 1 for the exponent $\gamma$. The decay time $\tau$ of the signal shows a large increase close to $T_{\mathrm{c}}$.

- close to the transition, the threshold may not follow a linear variation (see Fig. 23). Simultaneously the shape of the photo-signal shows a broad pulse following the peak due to the instability. This will be discussed in the next paragraphs ;

- for equally old samples the threshold dispersion is small. However this dispersion increases as the samples age.

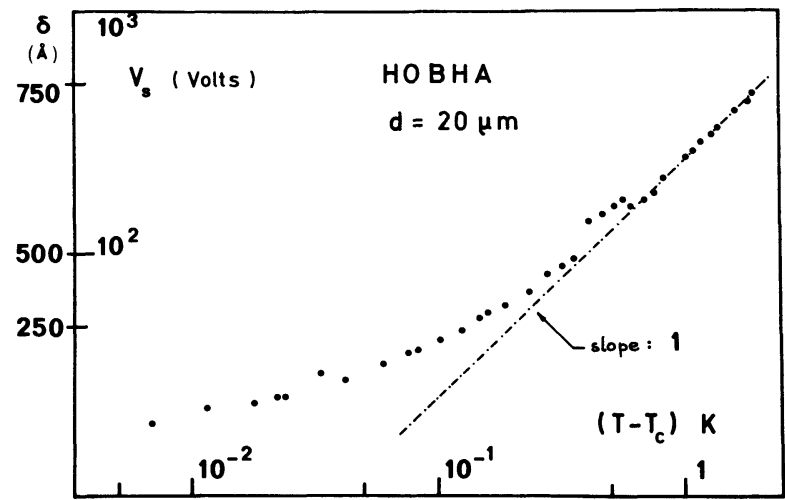

FIG. 23. - Temperature dependence of the voltage threshold $V_{\mathrm{S}}$ close to an $\mathrm{A} \rightarrow \mathrm{C}$ transition and in the presence of a hydrodynamic instability due to coupling between the molecular orientation and a unidirectional flow.

3.3.2 e) Temperature dependence of the decay time $\tau_{\mathrm{d}}$. The times $\tau_{\mathrm{d}}$ are measured at $10 \%$ above threshold. We observe that $\tau_{\mathrm{d}}$ increases as $T_{\mathrm{c}}$ is approached, as predicted in our simple model, the increase however being much larger. In fact the measurements become inaccurate close to $T_{\mathrm{c}}$ because there the signal decay departs more and more from a single exponential. We believe that, as for the undulation instability, the relaxation mechanism is not the unique one described in our simple model, and that each contribution has a different temperature dependence. We may suggest that far from $T_{\mathrm{c}}$ the simple dislocation motion is the dominant relaxation mechanism, while close to $T_{\mathrm{c}}$ another mechanism appears such as the hydrodynamic flow or the motion of large defects.

3.3.2 $f$ ) Observation of a structure deformation related to a unidirectional flow. - In some samples we have observed that the $V_{\mathrm{s}}=f\left(T-T_{\mathrm{c}}\right)$ curve shows a change of slope close to $T_{\mathrm{c}}\left(\Delta T \sim 5 \times 10^{-2}{ }^{\circ} \mathrm{C}\right)$. In that case the signal appears as the usual peak followed by a broad bell-shaped pulse (typical width : $300 \mathrm{~ms}$ ), see figure 24 . A very similar pulse may appear with a reverse sign for the dilation phase. As the voltage is increased the pulse height increases and it gets closer to the peak. Finally the two signals are not resolved and the final shape looks like a peak followed by a more or less exponential-like decay. The same evolution is observed with the voltage maintained constant and the temperature being varied towards $T_{\mathrm{c}}$.

Direct observation under the microscope in the same conditions reveals that for the compression phase the observed area lightens quickly (tilt instability), and morever a pattern of well-contrasted and equallyspaced straight lines appears. The spacing is of about 80-100 $\mu \mathrm{m}$ (for usual sample thickness of $20 \mu \mathrm{m}$ ). These lines appear suddenly, then move in a direction normal to their common alignment (and in a plane parallel to the glass plates). Their contrast decreases, and they disappear. Typically this deformation lasts for 100 to $500 \mathrm{~ms}$, and we have checked that it corres- 

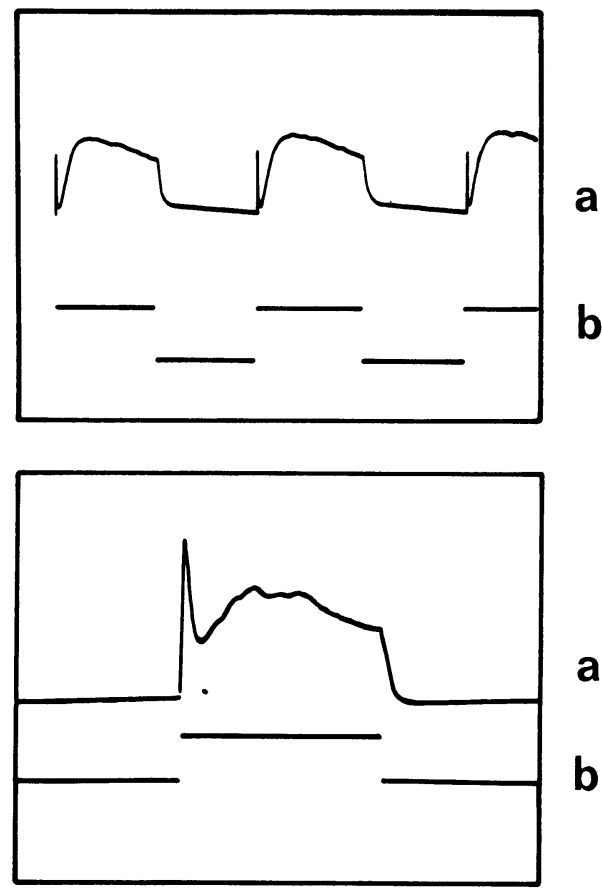

FIG. 24. - Shapes $(a)$ of the photo-signal due to the hydrodynamical instability (coupling between the flow and the molecular orientation). The first peak is due to the molecular tilt under compression. The square wave $(b)$ represents the excitation voltage.

ponds to the observation of the bell-shaped pulse. We observe also the same deformation during the dilation phase, at another place, having the same alignment but moving in the opposite direction. We check by removing the liquid crystal that the glass plates make a wedge and that the general direction of the lines was parallel to its edge. Generally this angle is found small, of the order $10^{-3} \mathrm{rad}$. The parallelism of the plates can be adjusted to a higher accuracy ( $\$ 10^{-4} \mathrm{rad}$ ).

We suggest that the imposed displacement of the glass induces a hydrodynamic flow with high velocities. and there may exist a coupling between the molecular orientation and the flow. This coupling would then produce the observed deformations which are thought to be undulations of the layers. When this coupling tends to reinforce the molecular tilt, the instability threshold decreases and vice-versa, which would explain the departure from the linear variation of the $V_{\mathrm{s}}=f\left(T-T_{\mathrm{c}}\right)$ curves (Fig. 23).

Finally, the existence of a very well-defined threshold under a compressive stress seems more compatible with a picture of a smectic-A phase in which the molecules are on the average normal to the plane of layers, rather than with a picture in which the molecules would be tilted inside each layer [28] (and randomly from one layer to other, the general orientation averaged over the whole sample resulting as normal to the layers).

3.3.2 g) Modulation of the molecular tilt angle in the smectic-C phase. - Applying sequences of compression-dilation in the smectic-A phase produces positive peaks of signal indicating an increase of transmitted light. Below the transition, we observe in the absence of any applied voltage a fluctuating weak signal that might be due to the thermal fluctuations corresponding to the twist mode [23], and the increase of the light level due to the molecular tilt, in the C phase. In this phase and close to $T_{\mathrm{c}}$ (typically $0.1^{\circ} \mathrm{C}$ ) we observe a symmetric signal (Fig. 25) that comes from the modulation of the molecular tilt angle around its position defined by the temperature. The amplitude of the peaks increases as the temperature decreases, being maximum in the middle of the smectic- $C$ range.

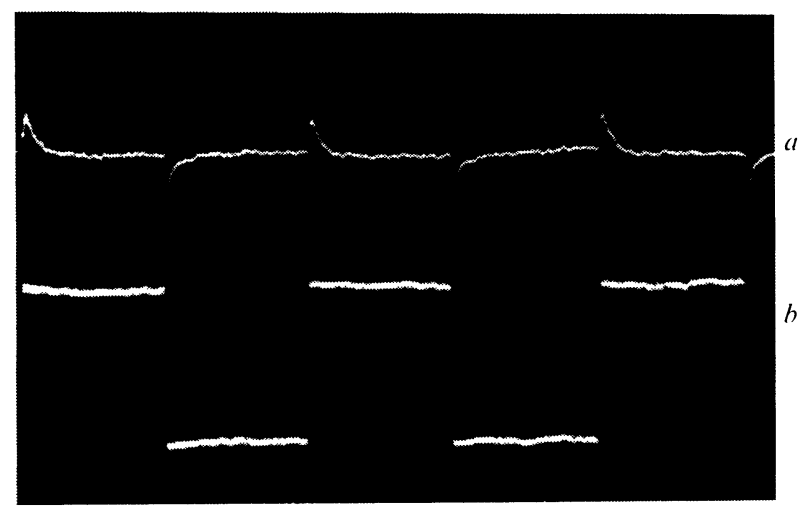

FIG. 25. - Photo-signal (a) due to the modulation of the tilted axis of molecules by the alternative sequences of compression dilation $(b)$ in the $C$ phase of HOBHA.

We have also noticed that in this phase the system is highly sensitive to mechanical vibrations imposed on the set-up. Small perturbations then produce large amplitude fluctuations of the optical signal.

3.3.2 h) Conclusion. - We have studied the molecular tilt instability under compressive stress, and close to a smectic-A to smectic-C transition. Our theoretical predictions are verified, in a transient regime : there exists a maximum pressure $B_{\perp}$ normal to the layers, beyond which a molecular buckling is observed. We have shown that the transverse rigidity measured by $B_{\perp}$ vanishes as the $\mathrm{A} \rightarrow \mathrm{C}$ transition is approached. The temperature dependence of the instability threshold is found quasi-linear in agreement with a molecular field theory. However this variation is found non-reproducible close to $T_{\mathrm{c}}$. Observation under the microscope reveals periodic structure deformations that may come from a coupling between the molecular tilt and a hydrodynamic flow. This coupling is much easier close to $T_{\mathrm{c}}$ and the deformations produced strongly affect the instability process. On a larger time scale, the molecular buckling disappears, the motion of defects relaxing the applied stresses.

As a practical application, the molecular tilt instability could be used to realize light modulators [24], or pressure transducers. The effect of modulation of the tilt angle in the $\mathrm{C}$ phase could give a method for measuring the temperature dependence of the tilt angle of the molecular axis. 
5. Conclusion. - In this paper we have given a theoretical treatment of the instabilities arising in a layered structure such as a smectic-A liquid crystal, under dilative and compressive stresses. When a dilative stress is applied normal to the layers an instability of structure appears on a short time scale as an undulation of well-defined wave-vector. We have studied experimentally the main features of this instability, and the most important result is the direct measurement of the de Gennes penetration length $\lambda$. Close to a quasi-second-order smectic-A to nematic transition $\lambda$ diverges as expected but with an apparent critical exponent which is too small. This result is not explained. On the other hand, close to a quasi-secondorder smectic-C phase transition the instability threshold decreases by a factor of almost two, as predicted by our model.

On a longer time scale, we observe the relaxation of the applied stresses normal to the layers, through the climb of dislocations or other defects. The observation seems to indicate the existence of two types of defects : the first ones relax the stress in a short time, the other ones come from the edges and correspond to larger times. The direct observation under the microscope of these defects allows us to give the first estimate of the mobility of edge dislocations in the smectic-A phase.

When a compressive stress is applied normal to the layers the molecular axis may tilt inside the layer above a well-defined threshold corresponding to a critical pressure $B_{\perp}$ normal to the layers. Experimentally it is easier to observe this instability close to a second-order smectic-A-smectic-C phase change. The temperature dependence shows that the threshold is decreasing as the $\mathrm{A} \rightarrow \mathrm{C}$ transition is approached, with a critical exponent close to one as would be expected from a mean-field theory. Close to the transition we have observed structural deformations that may alter the elastic response of the material and that are thought to arise from a coupling between the molecular orientation and the hydrodynamic flow produced by the applied stresses. As for the undulation instability, the molecular buckling instability appears only in a transient regime, the applied stresses relaxing rapidly through motion of defects.

During that transient regime, the observed phenomena are in reasonable agreement with the predictions of the theoretical part : existence of a defined displacement or stress threshold. and of a finite wave-number for the instability ; this regime can be called the elastic regime. For longer times (larger than a relaxation time of the order of $10 \mathrm{~ms}$ ) the smectic material cannot sustain the applied stresses normal to the layers. Internal readjustment of defects relaxes the stresses. We are in a plastic regime.

As a general conclusion, the initial model appears to be quite appropriate for describing and explaining the elastic behaviour of ideal smectics. However in order to explain the deformations of real smectics one must find methods of controlling the nature and the density of the defects which play a crucial role in the viscoelasticity of these phases. In that respect the case of smectics is quite reminiscent of that of real solids with a qualitative but important difference : the elastic response of the material is only visible within a very short time compared to the usual duration of an experiment. Beyond this time, plasticity becomes the dominant process for sample deformations. Models describing the possible role of defects have recently been developed by Kleman [29], and Prost and Pershan [30]. A new field of experimental investigations is open in that direction.

\section{References}

[1] Delaye, M., Ribotta, R., Durand, G., Phys. Lett. 44A (1973) 139.

[2] Clark, N. A., Meyer, R. B., Appl. Phys. Lett. 22 (1973) 10.

[3] Ribotta, R., Meyer, R. B., Durand, G., J. Physique Lett. 35 (1974) L-161.

[6] De Gennes, P. G., C. R. Hebd. Séan. Acad. Sci. 274B (1972)

- Also in The Physics of Liquid Crystals (Oxford Univ. Press) 1974.

[5] De Gennes, P. G., Solid State Commun. 10 (1972) 753.

- Mol. Liq. Crystals 21 (1973) 49.

[6] De Gennes, P. G., C. R. Hebd. Séan. Acad. Sci. 274 B (1972) 758.

[7] Durand, G., C. R. Hebd. Séan. Acad. Sci. 275B (1972) 629.

[8] McMillan, W. L., Phys. Rev. A 6 (1972) 936.

[9] Ribotta, R., J. Physique Colloq. 37 (1976) C3-149. (Proceedings of Les Arcs European Conference on Smectics, Déc. 1975.)

[10] Orsay Group on Liquid Crystals, J. Chem. Phys. 51 (1969) 816.

[11] Ribotta, R., Durand, G., Litster, D. L., Solid State Commun. 12 (1973) 27.

[12] Ribotta, R., Thèse Orsay, série A, no 1450 (1975), C.N.R.S. $n^{\circ}$ AO 11486

[13] Ribotra, R., in les Houches Lectures Notes (August 1973) Edited by R. Balian and G. Weill, Gordon \& Breach.

[14] Ribotta, R., Durand, G., 5th Int. Liquid Crystal Conference, Stockholm (1974).
[15] Ribotta, R., C. R. Hebd. Séan. Acad. Sci. 279B (1974) 295.

[16] Parodi, O., Solid State Commun. 11 (1972) 1503.

[17] Ribotta, R., Salin, D., Durand, G., Phys. Rev. Lett. 32 (1974) 6.

[18] Kleman, M., Friedel, J., J. Physique Colloq. 30 (1969) C4-43.

[19] Clark, N. A., Abstract of the Vth Int. Liquid Crystal Conf. Stockholm (1974).

[20] BiRECKI, H., SChaEtzing, R., Rondelez, F. and Litster, J. D., Phys. Rev. Lett. 36 (1976) 1376.

[21] Cladis, P. E., Phys. Rev. Lett. 31 (1973) 1200.

[22] SALIN, D., Thèse $3^{\mathrm{e}}$ cycle, Orsay (1974).

[23] Galerne, Y., Martinand, J. L., Durand, G., Veyssie, M., Phys. Rev. Lett. 29 (1971) 561.

[24] Bartolino, R., Bertolotti, M., Scudieri, F., Sliwinski, A., J. Appl. Phys. 46 (1975) 1928.

[25] De Gennes, P. G., Phys. Fluids 17 (1974) 1645.

[26] Brochard, F., J. Physique 34 (1973) 11 and thèse Orsay A. 1271 (1974).

[27] Delrieu, J. M., J. Chem. Phys. 60 (1974) 1081.

[28] De VRIES, A., Abstract of the Vth Int. Liquid Crystal Conference, Stockholm (1974).

[29] Kleman, M., J. Physique 35 (1974) 595.

Kleman, M., Williams, C. E., J. Physique Lett. 35 (1974) L-49.

[30] Prost, J. and Pershan, P. S., J. Appl. Phys. 46 (1975) 2343. 